\title{
Semiconductor lasers and integrated devices
}

\author{
P. Brosson \\ Alcatel, \\ Route de Nozay, \\ F-91460 Marcoussis, France \\ pbrosson@free.fr
}

\section{INTRODUCTION}

A semiconductor laser is a very compact light source $(0.2 \mathrm{~mm}$ long) in which electric current (a few $\mathrm{mA}$ ) is converted into coherent optical power with high efficiency. In 1970, the successful Continuous Wave (CW) operation [Alferov 70,Hayashi 70] of the Double-Heterostructure laser at room temperature coincided with the achievement of low loss $(1 \mathrm{~dB} / \mathrm{km})$ optical fiber [Kapron 70]. These high improvements of both laser and optical fiber have motivated a strong excitement worldwide. Today, high performances, and reliable semiconductor lasers are available for commercial high bit rate lightwave communication systems. Their Mean Time To Failure (MTTF) is 100 years, a tremendous improvement compared with 100 hours lifetime in 1972!

A wide range of new different types of semiconductor integrated photonic components [Erman 95,Erman 96], derived from the semiconductor laser, have been demonstrated in lightwave communication systems. They include integrated modulator (electro-absorption, Mach-Zehnder), tunable laser, Semiconductor Optical Amplifier (SOA), wavelength converter, Transmitter-Receiver Device (TRD), Vertical Cavity Surface Emitting Laser (VCSEL), demultiplexer, semiconductor laser pump source for Erbium Doped Fiber Amplifier... These integrated devices are under investigation at OPTO+ with already very exciting performances, or commercially available at Alcatel Optronics.

Keywords: Semiconductor laser, Integrated Laser electro-absorption Modulator, Integrated Laser MachZehnder Modulator, Semiconductor Optical Amplifier, Wavelenght converter, Wavelenght DeMultiplexer, Wavelenght selector, Transmitter Receiver Device, Wavelenght Division Multiplexing. 
In this tutorial, we will review some of these devices and discuss their principle of operation and applications. The tutorial will be organized as follows:

- Integrated Laser electro-absorption Modulator (ILM)

- Integrated Laser Mach-Zehnder Modulator (ILMZ)

- Semiconductor Optical Amplifier (SOA)

- Wavelength converter

- Wavelength DeMultiplexer (WDM)

- Wavelength selector

- Transmitter Receiver Device (TRD)

- Sources for Wavelength Division Multiplexing (WDM)

\section{INTEGRATED LASER ELECTRO-ABSORPTION MODULATOR (ILM)}

\subsection{Introduction}

At high modulation bit rates such as $10 \mathrm{~Gb} / \mathrm{s}$, due to optical frequency chirp in a current modulated laser, the transmission distance over a standard optical fiber is limited by the chromatic dispersion $\left(17 \mathrm{ps} . \mathrm{nm}^{-1} \cdot \mathrm{km}^{-1}\right.$ positive dispersion around $1550 \mathrm{~nm}$ wavelength). The optical frequency chirp in a current modulated laser is induced by the refractive index modulation which is due to carrier modulation. Close to the resonance frequency of the laser, the carrier modulation is enhanced and this leads to a strong optical frequency chirp (resonant chirp). This effect is an intrinsic behavior in current modulated lasers.

With standard optical fiber, at $1550 \mathrm{~nm}$, a chirp-less source is required to achieve bit rates higher than $10 \mathrm{~Gb} / \mathrm{s}$. It is expected that fast and chirp-less modulation could be achieved by using a cw laser coupled into an external modulator based on a fast physical effect such as electro-absorption. In this case, the resonant chirp is suppressed.

\subsection{The discrete electro-absorption (E/A) modulator}

By applying a reverse voltage $V$ on a p-i-n heterostructure (Fig. 1), due to the applied electric field $F$ in the intrinsic layer, the absorption spectrum (Fig. 2) is shifted towards low energy. The light emitted by a CW laser (DFB, wavelength $\lambda=1525 \mathrm{~nm}$ ) and coupled into the $E / A$ modulator is absorbed on application of a reverse voltage. The DFB laser and the $E / A$ modulator are designed such that at $\lambda=1550 \mathrm{~nm}$, the absorption is low for $V=V_{\text {on }}=0$ and high for $V=V_{\text {off }} \neq 0$. The extinction ratio of this modulator which is defined as the ratio of the output power $P$ between the two states is given by

$$
E R=\frac{I\left(V_{\text {on }}\right)}{I\left(V_{\text {off }}\right)}
$$




\section{reverse bias}

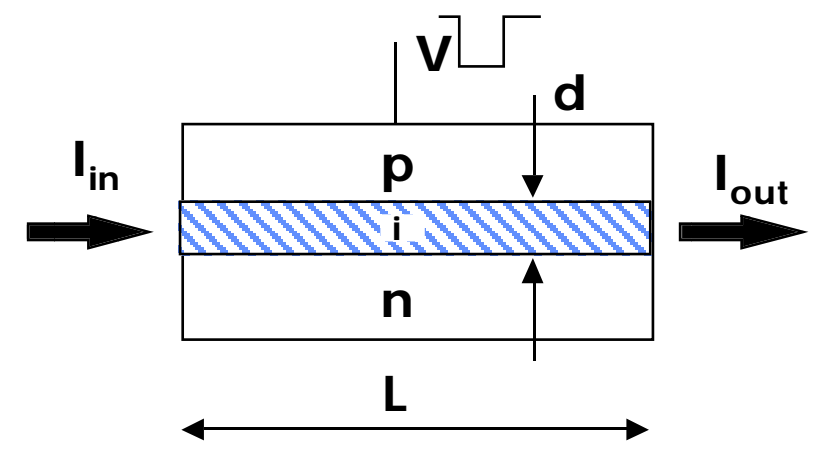

Figure 1. - Operating principle of the E/A modulator. CW light is injected in the modulator and absorbed in the intrinsic layer.

Defining $\Delta \alpha$ as the variation of the absorption between the two states, $\Gamma$ the optical confinement factor, the static extinction ratio is

$$
E R(\mathrm{~dB})=10 \log _{10}(e) \Gamma \Delta \alpha .
$$

Typical values are $E R=10 \mathrm{~dB}$ for $L=200 \mu \mathrm{m}$ and a peak to peak voltage modulation $V_{\mathrm{pp}}=1 \mathrm{~V}$.

A modulated light signal is obtained by modulating the voltage applied to the modulator. Unfortunately, the modulated optical signal at the modulator output is not completely chirp-less. The fast change of the optical absorption in the modulator induces a refractive index change that in turn induces a variation of the phase of the optical signal. This transient phase variation at the leading and falling edges of the driving pulse is traduced by a transient chirp. To describe this behavior, a phase amplitude parameter $\alpha_{\mathrm{H}}$ or chirp parameter (similar to the Henry factor in a laser) is defined

$$
\alpha_{\mathrm{H}}=\frac{\frac{\mathrm{d} n_{\text {real }}}{\mathrm{d} F}}{\frac{\mathrm{d} n_{\text {imag }}}{\mathrm{d} F}}=\frac{\frac{\mathrm{d} n_{\text {real }}}{\mathrm{d} V}}{\frac{\lambda}{4 \pi} \frac{\mathrm{d} \alpha}{\mathrm{d} V}}
$$

$n_{\text {real }}$ and $n_{\text {imag }}$ are the real and imaginary part of the refractive index.

In an $E / A$ modulator, the chirp parameter $\alpha_{\mathrm{H}}$ can be negative or positive, depending on the applied bias voltage and operating wavelength. In a laser, the chirp parameter is always positive. With a positive $\alpha_{\mathrm{H}}$, the leading edge of the light pulse presents a positive transient frequency chirp (blue frequency shift) and the falling edge a negative transient frequency chirp (red frequency shift). The optical pulse is broadening and narrowing along the fiber (Fig. 3) respectively for $\alpha_{\mathrm{H}}>0$ and $\alpha_{\mathrm{H}}<0$.

Using tensile strained Multi-Quantum Well (MQW) electro-absorption modulator and adjusting the reverse bias, the chirp parameter $\alpha_{\mathrm{H}}$ can be reduced to a value 


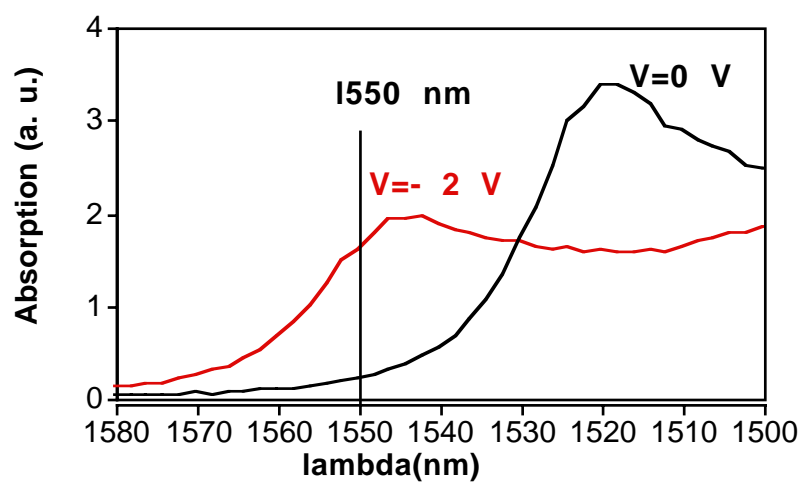

Figure 2. - Absorption spectrum of the E/A modulator. For $V=0 \mathrm{~V}$, the absorption is low at $1550 \mathrm{~nm}$. For $V=-2 \mathrm{~V}$, the absorption spectrum is shifted towards low energy and the absorption is high at $1550 \mathrm{~nm}$.

between 0 and -1 . This red transient chirp compensates the dispersion of the fiber, leading to a low chirp penalty. A $42 \mathrm{GHz}$ modulation bandwidth [Haisch 95] has been obtained with these modulators. However, the reverse bias, required to adjust the $\alpha_{\mathrm{H}}$ parameter, increases the optical loss on the "on" state, leading to a degradation of the extinction ratio. This inconvenient can be eliminated in a Mach-Zehnder modulator as described in the next section.

\subsection{The Integrated Laser E/A Modulator}

Since long transmission distance high bit rates with low penalty can be achieved using a discrete modulator, integration of the laser with the modulator (ILM) is attractive in order to reduce the cost (the packaging is highly simplified) and improve the optical output power (the coupling between the laser and modulator is intrinsically higher). However in an ILM, the optical feedback from the output facet of the modulator section becomes critical regarding the optical frequency chirp. Modeling and a careful design are required to minimize the chirp penalty.

\subsubsection{Modes and laser threshold of the ILM}

The schematic structure of the ILM is shown in Figure 4. Subscript 1 refers to the laser section and 2 to the modulator section. $n_{1}, n_{2}$ are the effective refractive indices, $\alpha_{2}$ the intensity optical modal loss of the modulator, $R_{1}=r_{1}^{2}, R_{2}=r_{2}^{2}$ the facet reflectivities of the laser and modulator, $L_{1}$ and $L_{2}$ their respective lengths, $I$ the current intensity in the laser and $V$ the applied voltage on the modulator section. The modulator output facet is AR coated (residual reflectivity $R_{2}<10^{-4}$ ) in order to avoid optical feedback into the laser section.

A multisection device such as an ILM can be described by the transmission line formalism [Tromborg 87], using the concept of effective reflectance. In the plane 


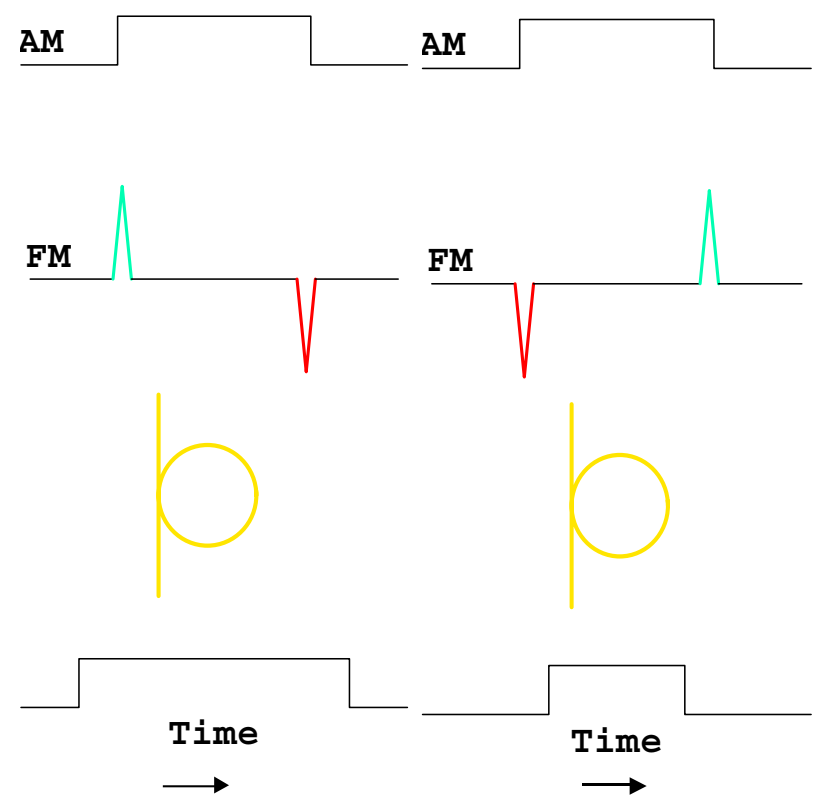

(a)

(b)

Figure 3. - (a) Frequency chirp at the output of a discrete E/A modulator $\left(\alpha_{\mathrm{H}}>0\right)$. At the leading edge of the voltage pulse, absorption drops and the refractive index decreases since $\alpha_{\mathrm{H}}>0$. The consequent transient phase variation is traduced by a transient optical frequency increase (blue shift). Opposite shift occurs at the falling edge of the pulse, leading to a transient optical frequency decrease (red shift). The chirped signal propagating in a standard fiber at $1550 \mathrm{~nm}$ is broadened since blue shifted component has a higher group velocity than red shifted component; (b) frequency chirp at the output of a discrete E/A modulator $\left(\alpha_{\mathrm{H}}<0\right)$. At the leading edge of the voltage pulse, absorption drops and the refractive index increases since $\alpha_{\mathrm{H}}<0$. The consequent transient phase variation is traduced by a transient optical frequency decrease (red shift). Opposite shift occurs at the falling edge of the pulse, leading to a transient optical frequency increase (blue shift). The chirped signal propagating in a standard fiber at $1550 \mathrm{~nm}$ is narrowed since blue shifted component has a higher group velocity than red shifted component.

separating the laser and modulator sections, the left and right effective reflectances are defined as $r_{\mathrm{L}}=\frac{E^{+}(0)}{E^{-}(0)}$ and $r_{\mathrm{R}}=\frac{E^{-}(0)}{E^{+}(0)}$ where $E^{+}$and $E^{-}$are the complex optical field components propagating in the right and left directions.

Continuity of the optical field imposes the condition (laser threshold condition)

$$
r_{\mathrm{L}}(\omega, N) r_{\mathrm{R}}(\omega, V)=1
$$

where $\omega$ is the optical frequency and $N$ the threshold carrier density. The grating coupling coefficient $K$ of the DFB laser section describes the amount of distributed feedback between $E^{+}$and $E^{-}$in the DFB section. 


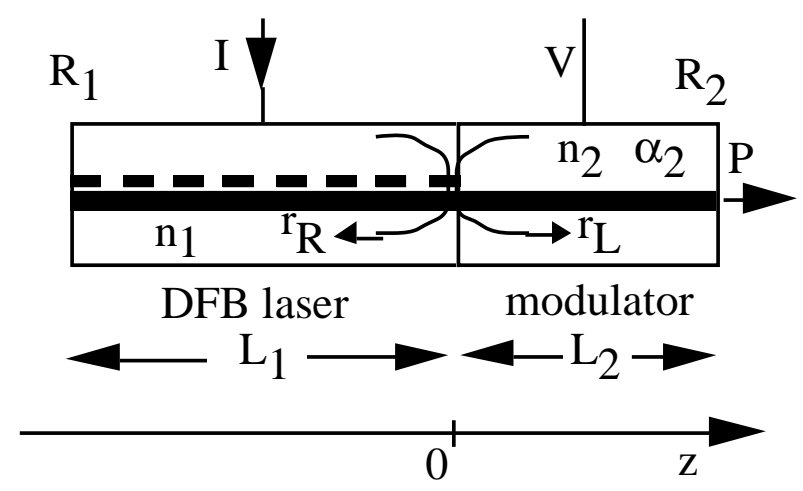

Figure 4. Schematic representation of the Integrated DFB Laser Modulator (ILM).

In DFB lasers, the position of the grating relative to the output facets strongly influences the laser threshold and the optical spectrum. $\varphi$ is the phase of the modulator output facet relative to the grating. The resolution of the complex oscillation condition (Eq. 4) gives the DFB modes (optical frequency $\omega$ ), their corresponding threshold gain (threshold carrier density) and the modal selectivity (threshold gain difference between main and satellite modes). The threshold current is slightly sensitive to change of $L_{1}$ or $K$, however the external efficiency can be improved (increased output power) by reducing $L_{1}$ or $K$. An example of optimization is the increased output power up to $20 \mathrm{~mW}$ by reducing the DFB coupling coefficient $\left(60\right.$ to $\left.40 \mathrm{~cm}^{-1}\right)$ which has allowed $76 \mathrm{~km}$ transmission at $10 \mathrm{~Gb} / \mathrm{s}$ over dispersive standard fiber without any optical amplifier [Lesterlin 97].

\subsubsection{Dynamic response of the ILM}

Since the main difficulty in this device is to control the frequency chirp, we shall focus on this aspect, and will not discuss in detail modulation bandwidth degradation due to electrical parasitic (capacitance).

A modulated reverse voltage is applied to the modulator section and its varying absorption modulates the light output of the laser which is biased above threshold. With $R_{2}>2 \times 10^{-4}$, a frequency chirp is observed in this integrated device (Fig. 5). The optical signal at the output facet of the modulator is oscillating as well as the optical frequency. The transient chirp at the leading (red shift) and falling (blue shift) edges of the driving pulse is attributed to the modulator section. The frequency difference $\Delta f_{\text {adiab }}=f_{\text {on }}-f_{\text {off }}$ between the "on" and "off" states is called adiabatic chirp. Theoretical models [Brosson 96] of DFB lasers show that $\Delta f_{\text {adiab }}$ depends on the phase $\varphi$ at the facets of the ILM. Since cleaving of the devices cannot be controlled better than a few micrometers, the phase is random and consequently the sign of $\Delta f_{\text {adiab }}$ is random too. Inside the pulse, the power and optical frequency oscillations at the resonance frequency of the laser are attributed to the optical feedback from the output facet of the modulator. 


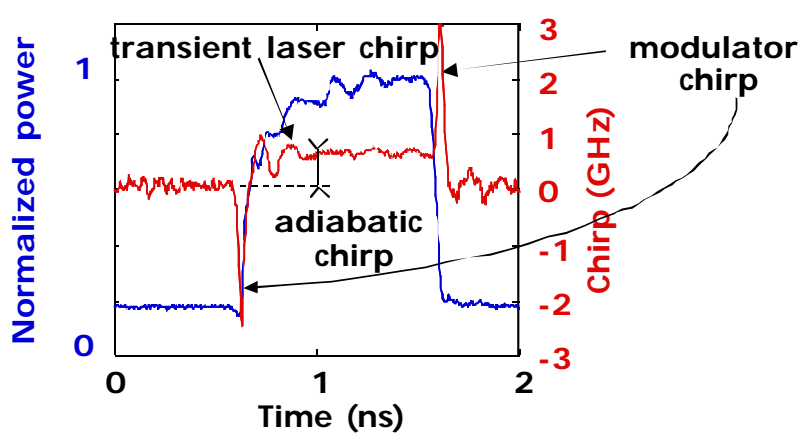

Figure 5. - Optical frequency chirp under digital modulation of an Integrated electroabsorption Laser Modulator.

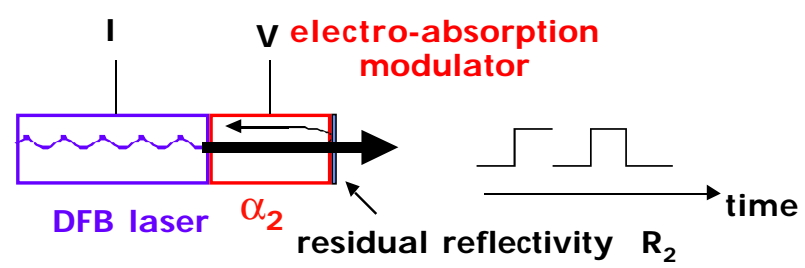

Figure 6. Origin of the feedback induced chirp in an Integrated Laser Modulator.

The frequency chirp originates [Brosson 96] from two effects (Fig. 6):

1) due to the optical feedback, the modulation of the optical loss of the modulator induces a change of the threshold carrier density of the laser and consequently a variation of the carrier density $N$. This leads to a modulation of $n_{1}$ and of the lasing wavelength.

2) Simultaneously, the modulation of $\alpha_{2}$ induces a change of the effective refractive index $n_{2}$, leading to a change of the phase of the light at the modulator output facet. This contributes to an additional optical frequency change, resulting in blue/red-shifted leading edge and red/blue-shifted falling edge of the pulse under digital modulation. This effective refractive index change is described by the modulator chirp parameter $\alpha_{\mathrm{H} 2}=\frac{\mathrm{d} n_{2}}{\mathrm{~d} V} /\left(\frac{\lambda}{4 \pi} \frac{\mathrm{d} \alpha_{2}}{\mathrm{~d} V}\right)$.

Even with a zero modulator reflectivity, this last contribution remains (Fig. 3). For modulation frequencies $f_{\text {mod }}$ close to the resonance frequency of the laser, the modulation of the carrier density is higher and the optical frequency oscillations are enhanced (resonant chirp). This frequency chirp is strongly dependent on the reflectivity $R_{2}$ and the facet phase. This dependence with $R_{2}$ is demonstrated in Figure 7 where experimental results [Lestra 96a] of the normalized FM to AM ratio $\beta / m=\frac{\delta f}{f_{\text {mod }}} / \frac{\delta P}{P}$ versus the modulation frequency are presented. Severe AR coating is expected to be required in order to minimize chirp in an ILM. 


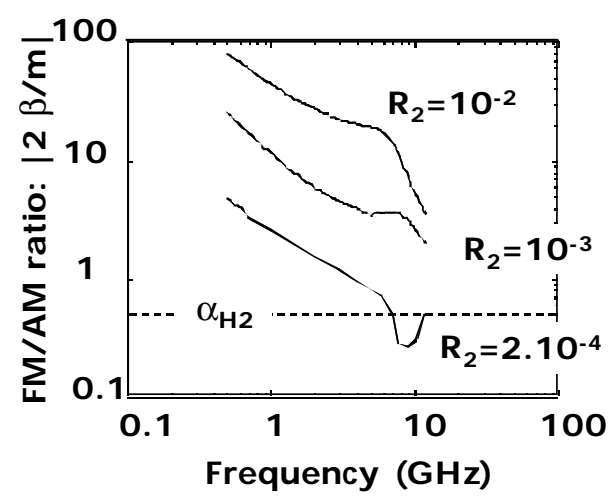

Figure 7. - Measured normalized FM/AM response: influence of the modulator facet reflectivity [Lestra 96a].
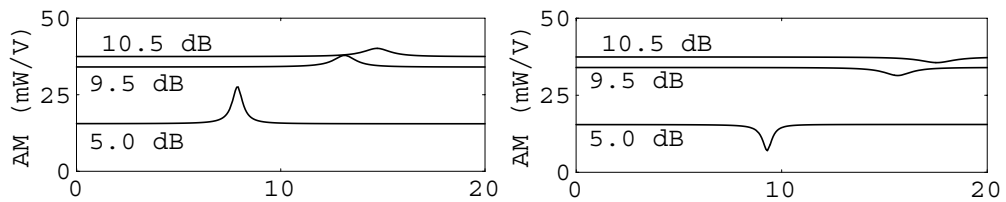

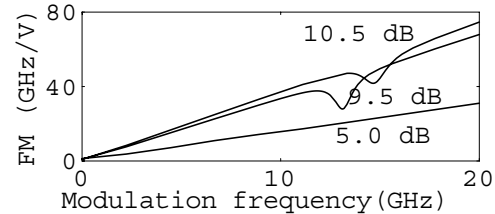

(a)

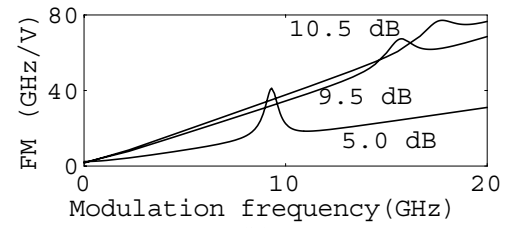

(b)

Figure 8. - (a) Amplitude and frequency modulation responses of the ILM at $P=5 \mathrm{~mW}$ for $\alpha_{2}=5,9.5,10.5 \mathrm{~dB}, \varphi=0.1 \pi$; (b) Amplitude and frequency modulation responses of the ILM at $P=5 \mathrm{~mW}$ for $\alpha_{2}=5,9.5,10.5 \mathrm{~dB}, \varphi=0.6 \pi$.

Small signal modulation

By solving the coupled rate equations in a small signal approximation, analytical expressions of the FM and AM responses have been obtained [Brosson 96]. The model allows to calculate, as a function of the modulation frequency $\Omega / 2 \pi$, (1) the AM response $\delta P / \delta V$, (2) the FM response $\delta f / \delta V$, (3) the normalized FM/AM $(2 \beta / m)$ response, $(4)$ the phase difference between FM and AM, (5) the carrier density modulation $\delta N / \delta V$.

Typical responses $\delta P / \delta V$ and $\delta f / \delta V$ are shown in Figure 8a. The peak observed near $16 \mathrm{GHz}$ corresponds to the resonance frequency of the laser. The behavior of these frequency responses is dependent on the phase $\varphi$ and consequently random. Typical responses for another value of $\varphi$ are shown in Figure 8b. The AM response 


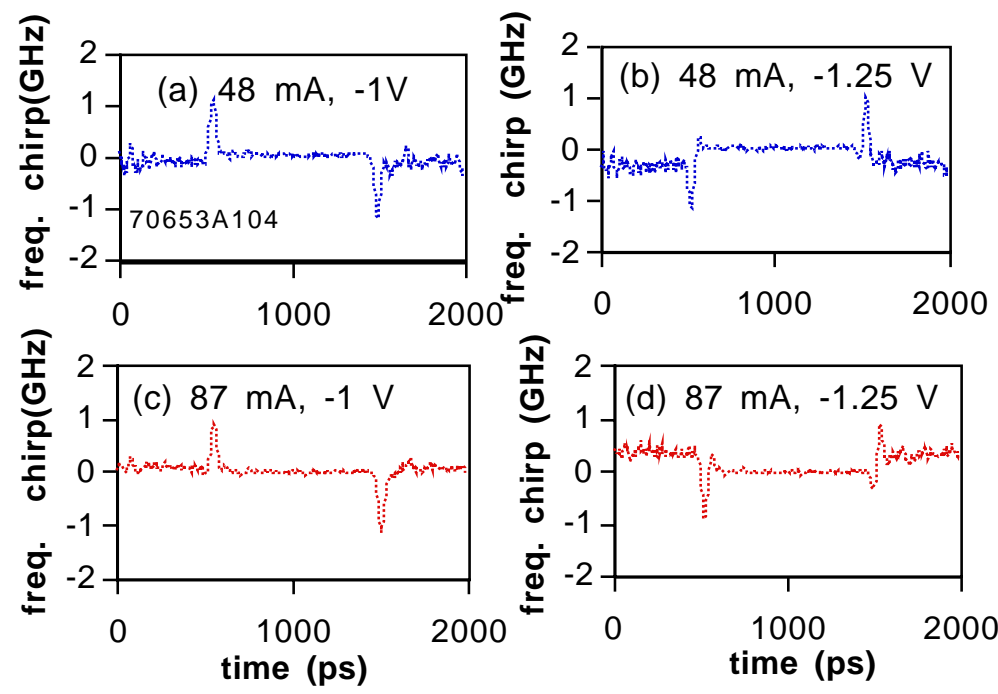

Figure 9. - Measured frequency chirp, (a) $48 \mathrm{~mA},-1 \mathrm{~V}$, (b) $48 \mathrm{~mA},-1.25 \mathrm{~V}$, (c) $87 \mathrm{~mA},-1 \mathrm{~V}$, (d) $87 \mathrm{~mA},-1.25$ V [J. G. Provost, private communication].

can be enhanced or depleted around the resonance frequency, depending on the value of the phase $\varphi$. In case (a) the value of the phase is such that the contribution of the modulator is in phase with that of the laser, in case (b), they are out of phase. The same analysis explains the depleted or enhanced FM response shown in Figure 7. This unusual behavior is actually in agreement with measured frequency responses.

\section{Digital modulation}

By numerically integrating [Brosson 97] the coupled rate equations, the responses to a pulse or a 16-bit pseudo random bit NRZ sequence at $10 \mathrm{~Gb} / \mathrm{s}$ is obtained. As in the case of small signal modulation, we calculate the time response of (1) the modulated output power $P(t),(2)$ the optical frequency $\delta f(t)$, (3) the phase of the optical signal $\Phi(t)$. Adiabatic chirp (variation of the optical frequency between the "on" and "off" states) depends on the phase $\varphi$ and therefore is highly random. Figure 9 shows the measured time response to a pulse at $1 \mathrm{~Gb} / \mathrm{s}$. Figures $9 \mathrm{a}$ and $9 \mathrm{~b}$ correspond to a DC current in the laser section of $48 \mathrm{~mA}$ and Figures $9 \mathrm{c}$ and $9 \mathrm{~d}$ to a current of $87 \mathrm{~mA}$. The bias voltages are -1 , and $-1.25 \mathrm{~V}$. These measurements clearly show that the adiabatic chirp can be positive (Figs. 9a, 9b) or negative (Fig. 9d), depending on the value of the DC current in the laser section. By changing the current, due to heating effects, the lasing wavelength changes, resulting in a change of $n_{2}$ and consequently of the phase $\varphi$. By increasing the applied bias voltage, $\alpha_{\mathrm{H} 2}$ changes from positive to negative, resulting in blue-shifted leading edge of the pulse (Figs. 9a, 9c) and redshifted (Figs. 9b, 9d). We also have observed a random behavior [Lestra 96a] of the chirp for $R_{2}=10^{-2}$, on devices from the same wafer, due to the random character of the laser facet phase. 

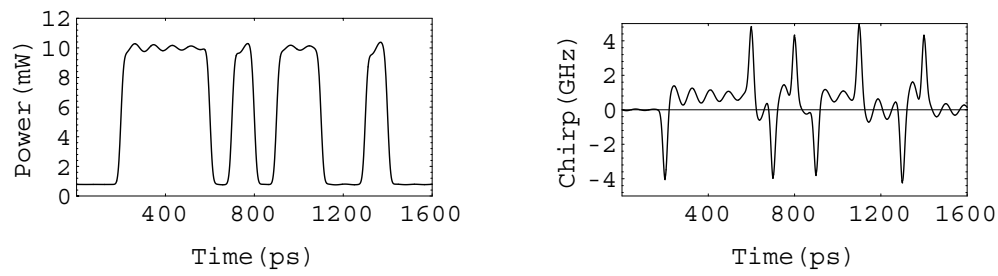

Figure 10. - Time response of the modulator modulated by a 16-bit NRZ sequence at $10 \mathrm{~Gb} / \mathrm{s}$ for a phase $\varphi=1.8$ rad., leading to a maximum positive adiabatic chirp; $\alpha_{\mathrm{H} 2}=-0.5$. (Left) Modulator output power; (right) Optical frequency chirp.
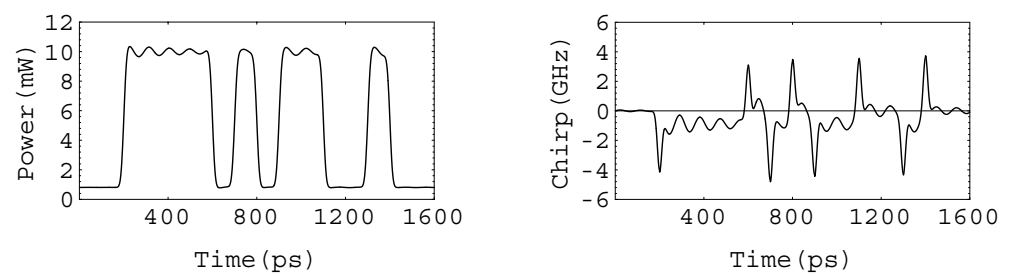

Figure 11. - Time response of the modulator modulated by a 16-bit NRZ sequence at $10 \mathrm{~Gb} / \mathrm{s}$ for a phase $\varphi=0.8 \mathrm{rad}$., leading to a maximum negative adiabatic chirp; $\alpha_{\mathrm{H} 2}=-0.5$. (Left) Modulator output power; (right) Optical frequency chirp.
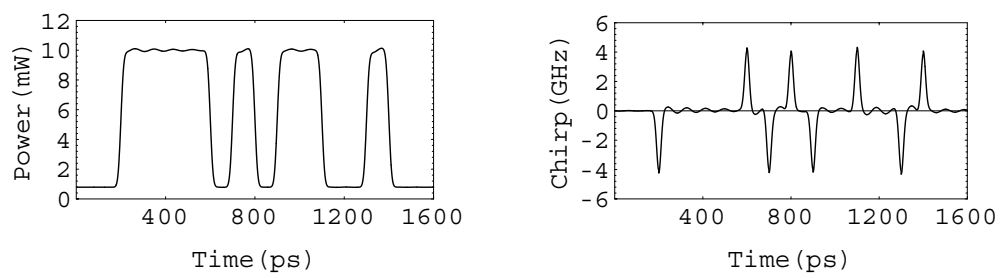

Figure 12. - Time response of the modulator modulated by a 16-bit NRZ sequence at $10 \mathrm{~Gb} / \mathrm{s}$ for a phase $\varphi=1.4$ rad., leading to zero adiabatic chirp; $\alpha_{\mathrm{H} 2}=-0.5$. (Left) Modulator output power; (right) Optical frequency chirp. 


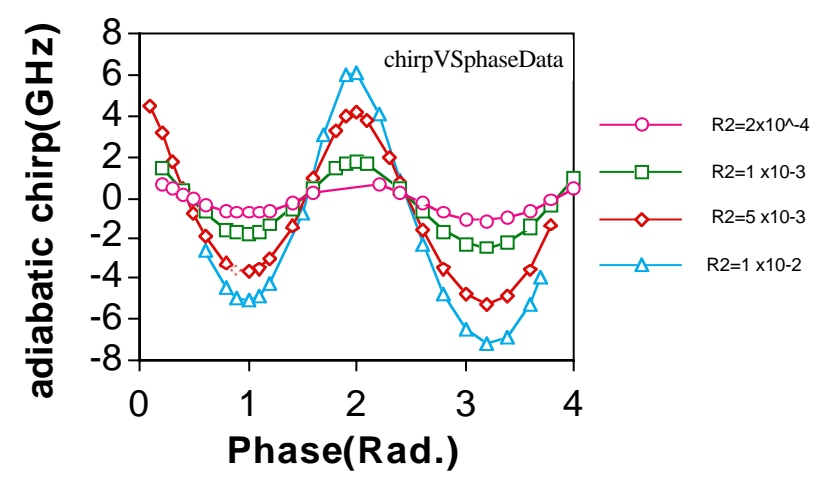

Figure 13. - Adiabatic chirp as a function of the phase $\varphi$ for a modulator facet reflectivity. $R_{2}=2 \times 10^{-4}, 1 \times 10^{-3}, 5 \times 10^{-3}, 1 \times 10^{-2}$. The chirp parameter of the modulator section is $\alpha_{\mathrm{H} 2}=0$.

We have investigated the effect of the phase $\varphi$ on the response to a 16-bit pseudo random bit NRZ sequence at $10 \mathrm{~Gb} / \mathrm{s}$ with a $11 \mathrm{~dB}$ extinction ratio. Typical theoretical responses are shown in Figures 10-12 for $\varphi=1.8,0.8$ and 1.4 rad., which correspond respectively to a maximum positive adiabatic chirp (blue shift), maximum negative adiabatic chirp (red shift), and zero adiabatic chirp. The order of magnitude of the chirp given by this numerical simulation is in agreement with time resolved chirp measurements at $1 \mathrm{~Gb} / \mathrm{s}$ (Fig. 9). The transient oscillations of the output power $P(t)$ (Figs. 10-12, left) and of the optical frequency $\delta f(t)$ (Figs. 10-12, right) correspond to the resonance frequency of the laser while the transient frequency chirp at the leading and falling edges of the pulses is due to the modulator section itself. The adiabatic chirp has been calculated (Fig. 13) as a function of $\varphi$ for $R_{2}=2 \times 10^{-4}, 1 \times 10^{-3}, 5 \times 10^{-3}$, $1 \times 10^{-2}$ for $\alpha_{\mathrm{H} 2}=0$. It is a periodic function of $\varphi$, whose amplitude depends on the value of the residual reflectivity $R_{2}$ of the modulator facet. By decreasing the residual reflectivity $R_{2}$, the adiabatic chirp decreases. Similar results have been obtained for $\alpha_{\mathrm{H} 2}=-1$.

\subsection{Conclusion}

Fast and low chirp modulation can be achieved with a good design ILM. Feedback induced chirp depends on the modulator facet reflectivity and phase. Despite the random character of the facet phase in an ILM, the adiabatic chirp can be $\leq 1 \mathrm{GHz}$ whatever the value of the phase $\varphi$, provided [Brosson 96] that the modulator facet reflectivity is $\leq 2 \times 10^{-4}$.

Owing to their improved packaging and performances, integrated DFB Laser Modulators (Fig. 14) are widely used for $10 \mathrm{~Gb} / \mathrm{s}$ transmission in standard fibers [Lesterlin 96b]. The modulator section consists of compressive strained MQWs ensuring low chirp parameter and low (1 volt) driving voltage. The laser section consists of compressive strained MQWs with $20 \mathrm{~mA}$ threshold current. The low capacitance required for high electrical bandwidth is obtained using a short ridge structure for 


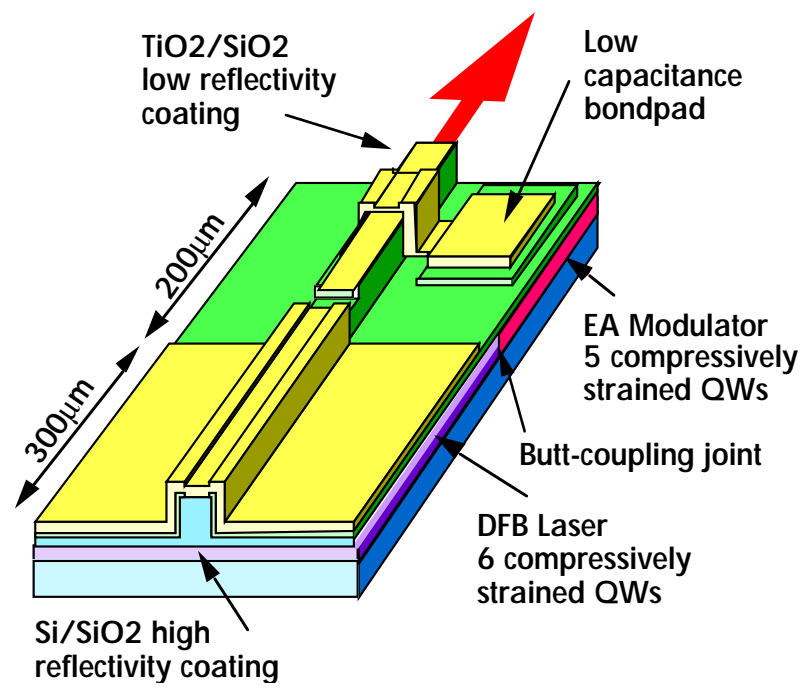

Figure 14. Integrated electro-absorption DFB Laser Modulator.

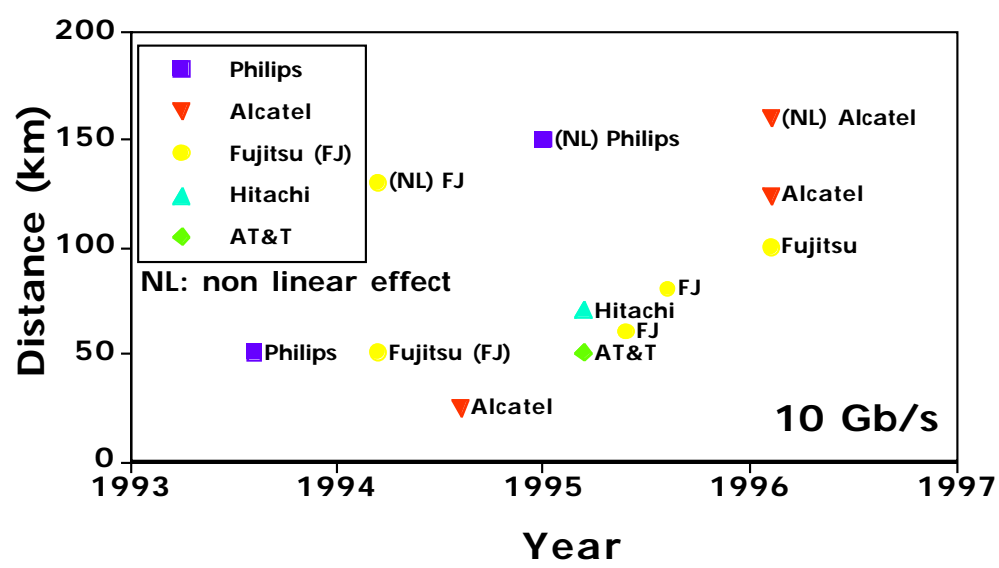

Figure 15. Integrated Laser Modulator at $10 \mathrm{~Gb} / \mathrm{s}$, state of the art (NL: non linear regime).

the modulator section $\left(L_{2}=200 \mu \mathrm{m}\right)$. Figure 15 shows the evolution of the state of the art for this device. The transmission distance was $\leq 50 \mathrm{~km}$ in 1993 and has reached $120 \mathrm{~km}$ these last years. 


\section{INTEGRATED LASER MACH-ZEHNDER MODULATOR (ILMZ)}

\subsection{Introduction}

In high bit rate optical links using conventional fiber, the required low frequency chirp of the transmitter can be obtained by using an external modulator, of electroabsorption [Haisch 95] or Mach-Zehnder [Delansay 97] type. Another attractive feature of the Mach-Zehnder modulator is its intrinsic wavelength independence with application in WDM systems.

\subsection{The discrete Mach-Zehnder interferometer modulator}

The schematic structure of the Mach-Zehnder modulator is shown in Figure 16. Both arms A and B consist of identical optical waveguides ( $\mathrm{p}-\mathrm{i}-\mathrm{n}$ heterostructure similar to the $E / A$ one) of length $L$. In contrast with an electro-absorption modulator, both arms are transparent at the operating wavelength $(1550 \mathrm{~nm})$. By applying reverse voltage $V$ on one arm (Fig. 17), at the operating wavelength, the absorption is slightly increased and a refractive index is change is induced.

The light emitted by a CW laser (DFB, wavelength $\lambda=1550 \mathrm{~nm}$ ) is coupled into the Mach-Zehnder interferometer. For $V=0, n_{\mathrm{A}}=n_{\mathrm{B}}$ and the light output is maximum at the operating wavelength. Applying a reverse voltage on arm A results in $n_{\mathrm{A}} \neq n_{\mathrm{B}}\left(n_{\mathrm{A}}-n_{\mathrm{B}} \cong 0.01\right)$ at the operating wavelength. If the optical path difference is an integer number $q$ of wavelength ( 0 phase difference), interferences of the optical signal propagating in both arms are constructive and the light output is maximum. The conditions read

$$
\left(n_{\mathrm{A}}-n_{\mathrm{B}}\right) L=q \lambda .
$$

Destructive interferences are obtained for $V=V_{\pi}$, with

$$
\left(n_{\mathrm{A}}-n_{\mathrm{B}}\right) L=q \frac{\lambda}{2}
$$

where $q$ is an integer.

Equation (5) is equivalent to

$$
\varphi_{\mathrm{A}}-\varphi_{\mathrm{B}}=q(2 \pi), \quad \text { with } \varphi_{\mathrm{A}, \mathrm{B}}=-\frac{2 \pi L}{\lambda} n_{\mathrm{A}, \mathrm{B}}
$$

assuming that the optical field is of the form $\exp (i \omega t-i \beta z)$. The lengths of both arms $\mathrm{A}$ and $\mathrm{B}$ can be equal (symmetric Mach-Zehnder) or intentionally mismatched by $\Delta L=L_{\mathrm{A}}-L_{\mathrm{B}}$ in order to obtain $\pi$-phase-shifted [Yu 96] Mach-Zehnder modulator at zero bias. The relation between $\Delta L$ and the phase difference $\Delta \varphi_{0}$ between both arms is given by $\Delta L=\frac{\lambda \Delta \varphi_{0}}{2 \pi n_{2}}$. If a modulation voltage $(10 \mathrm{~Gb} / \mathrm{s})$ is applied on electrode A (Fig. 18), the light output follows the modulation drive voltage. In the case of $\pi$-phase-shifted Mach-Zehnder modulator, an opposite signal is obtained. 


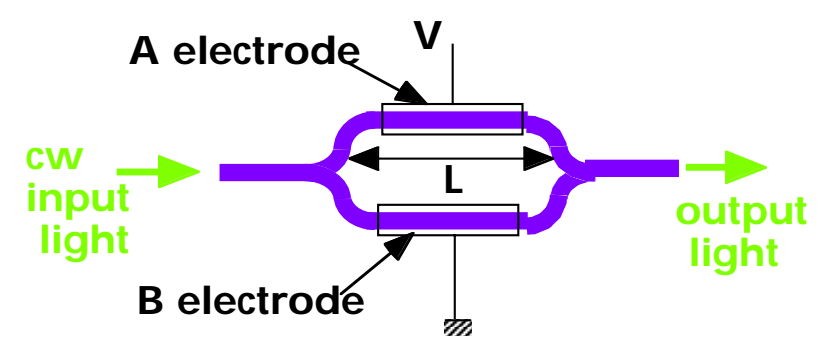

Figure 16. The discrete Mach-Zehnder interferometer modulator: operating principle.

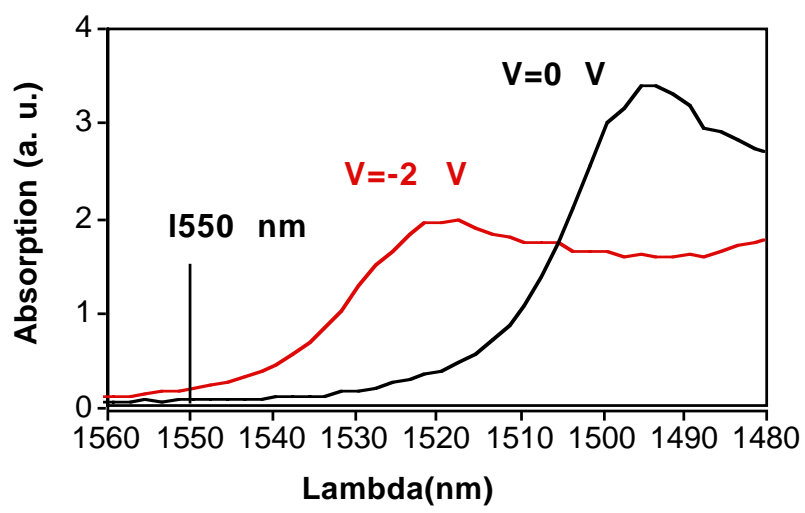

Figure 17. - Absorption spectrum of the Mach-Zehnder waveguide arm. For $V=0 \mathrm{~V}$, the absorption is low at $1550 \mathrm{~nm}$. For $V=-2 \mathrm{~V}$, due to the applied electric field $F$ in the intrinsic layer, the absorption spectrum is shifted towards low energy. At $1550 \mathrm{~nm}$ the absorption is still low but the refractive index is changed.

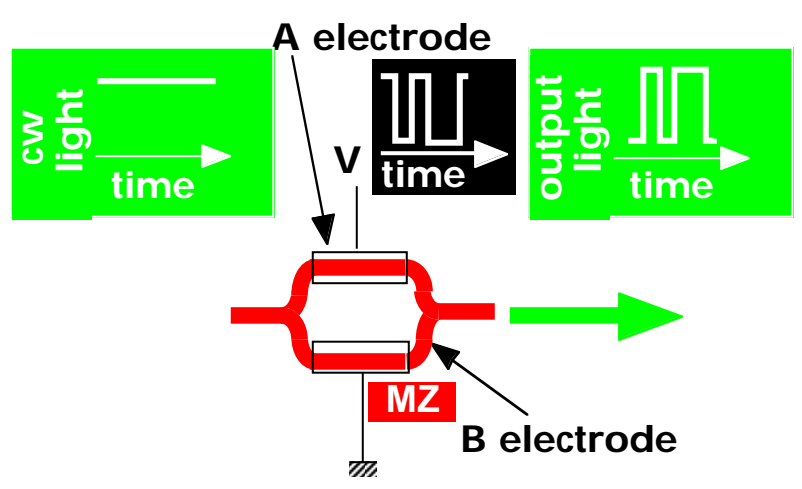

Figure 18. Discrete symmetric Mach-Zehnder modulator. 


\subsection{The Integrated Laser Mach-Zehnder modulator (ILMZ)}

\subsubsection{Introduction}

Integration of the laser with Mach-Zehnder [Delansay 98, Zucker 92, Tanbun-Ek 95, Adams 96] modulators are being extensively investigated to take advantage of their cost effective packaging.

However in an Integrated Laser Modulator, the residual optical feedback from the output modulator facet induces frequency chirp and relaxation oscillations which limit the transmission distance in high bit rate fiber optic links.

This has been observed in the case of Integrated Laser electro-absorption Modulators (ILM), which we have theoretically investigated [Brosson 96, Lestra 96b, Brosson 97]. For this type of modulator, the chirp parameter $\alpha_{\mathrm{H}}$ can be set [Lesterlin 96b] negative, close to zero, corresponding to an optimal transmission on a standard fiber, by adjusting the bias voltage of the modulator section. However, this leads to an increase of the optical loss.

In the case of the Integrated Laser Mach-Zehnder modulator with a $\pi$-phaseshifted configuration [Penninckx 97], the chirp is negative from zero to $V_{\pi}$ and close to -1 , thus allowing optimized driving conditions while keeping a reasonable high output power. Furthermore, the wavelength independent behavior of the $\pi$-phaseshifted configuration makes it an ideal modulator candidate for WDM oriented systems [Delansay 97].

Feedback effects have been investigated theoretically [Brosson 98b] deriving analytical expressions of the FM and AM responses, since FM response is an indicator of the chirp induced by optical feedback. The analysis allows identifying the direct and indirect contributions to the FM response.

\subsubsection{Modes and laser threshold of the ILMZ}

The schematic structure of the ILMZ and details of the waveguide are shown in Figure 19. Two Mach-Zehnder configurations are shown in Figure 20. In the first one $(2 \times 1$ output coupler), both arms of the Mach-Zehnder are coupled into a unique output waveguide. In the second one $(2 \times 2$ output coupler $)$, both arms are coupled into two output waveguides.

The integrated structure consists of two sections, the DFB laser and the MachZehnder. Subscript 1 refers to the laser section and 2 to the modulator section with arms $\mathrm{A}$ and B. $n_{1}, n_{2 \mathrm{~A}}, n_{2 \mathrm{~B}}$ are the effective refractive indexes, $\alpha_{\mathrm{A}}$ and $\alpha_{\mathrm{B}}$ the intensity optical modal loss, $L_{1}, L_{\text {in }}, L_{\text {out }}, L_{\mathrm{e}}=L_{\text {in }}+L_{\text {out }}, L_{\mathrm{A}}, L_{\mathrm{B}}$ the respective lengths (Fig. 20), $r_{1}=\sqrt{ } R_{1}, r_{2 \mathrm{~A}}=\sqrt{ } R_{2 \mathrm{~A}}, r_{2 \mathrm{~B}}=\sqrt{ } R_{2 \mathrm{~B}}, r_{2}=\sqrt{ } R_{2}$, the facet reflectivities (amplitude and intensity) of the laser and modulator, $I$ the current intensity in the laser and $V$ the reverse applied voltage on arm A of the modulator section.

$\kappa$ is the grating coupling coefficient of the DFB laser section, $\varphi_{1}$ and $\varphi_{2}$ the arbitrary phases of the left and right facets relative to the grating and $\alpha_{\mathrm{H} 2}=\frac{\mathrm{d} n_{2}}{\mathrm{~d} V} /\left(\frac{\lambda}{4 \pi} \frac{\mathrm{d} \alpha_{2}}{\mathrm{~d} V}\right)$ the chirp parameter of the Mach-Zehnder waveguides. A coupling ratio 50/50 between both waveguides with Multi-Mode Interference (MMI) couplers is assumed and the experimental extra loss $(0.4 \mathrm{~dB})$ coming from these couplers can been neglected. 


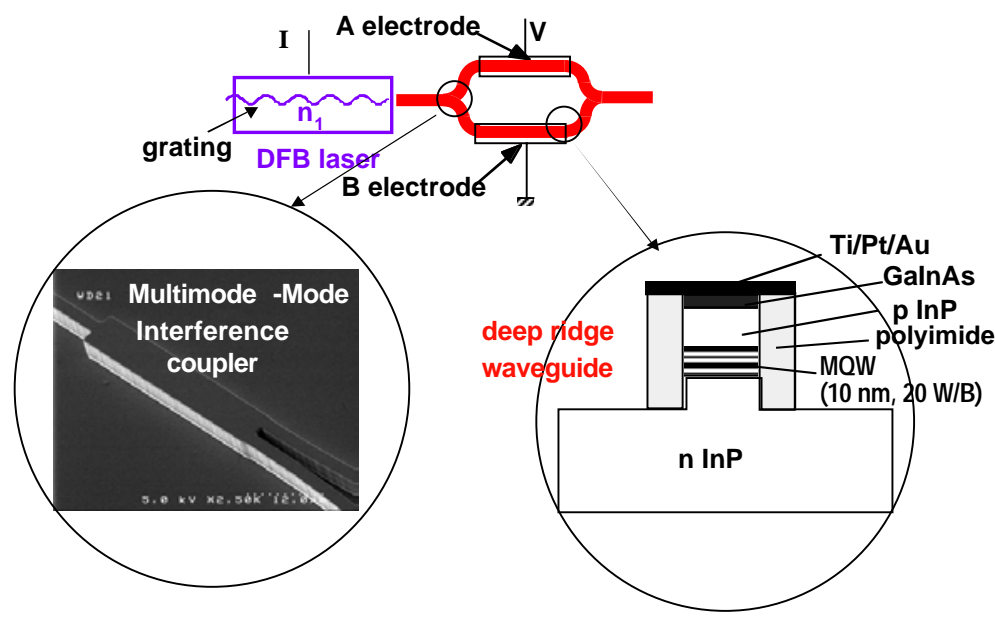

Figure 19. Integrated Laser Mach-Zehnder (ILMZ) modulator: waveguide structure.

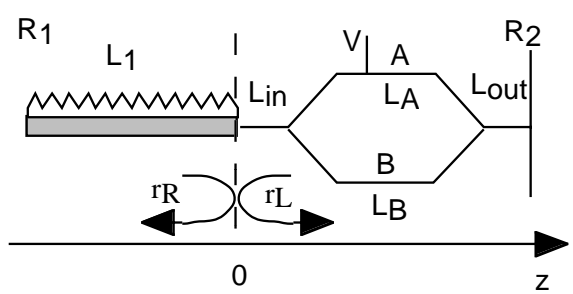

(a) 2x1 Mach-Zehnder

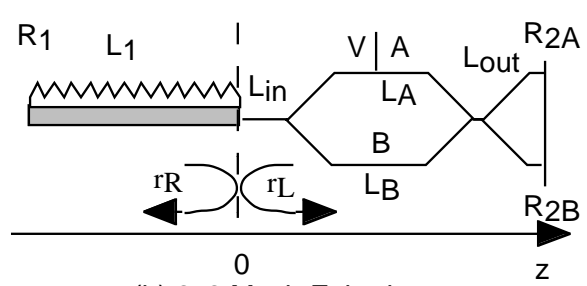

(b) 2x2 Mach-Zehnder

Figure 20. - Schematic representation of the Integrated DFB Laser Mach-Zehnder modulator (ILMZ). The reference plane $z=0$ is at the interface between the laser and modulator sections.

Using the same approach [Brosson 96] as for the ILM, we define $r_{L}, r_{R}$ the left and right complex effective reflectances of the DFB laser section and of the modulator section. The DFB laser and the Mach-Zehnder modulator are designed such that at $\lambda=1550 \mathrm{~nm}$, the absorption is low for both $V=V_{\text {on }}=0$ and $V=V_{\text {off }} \neq 0$ (Fig. 17). The modulator output facet is AR coated (residual reflectivity $R_{2}<10^{-4}$ ) in order to avoid optical feedback into the laser section.

In the configuration $2 \times 1$, the required additional phase for $\pi$-phase-shifted behavior is $\Delta \varphi_{0}=\pi$. In the configuration $2 \times 2$, the MMI coupler itself introduces a $\pi / 2$ phase shift, and the required additional phase for $\pi$-phase-shifted behavior is $\Delta \varphi_{0}=\pi / 2$. With $\lambda=1550 \mathrm{~nm}$ and $n_{2}=3.23, \Delta L=0.24$ and $0.12 \mu \mathrm{m}$ for the $2 \times 1$ and $2 \times 2$ Mach-Zehnder configurations respectively. The resolution of the complex oscillation condition $r_{\mathrm{L}}(\omega, N) r_{\mathrm{R}}(\omega, V)=1$ gives the DFB modes (optical frequency $\omega$ ) and their corresponding threshold gain (threshold carrier density $N_{\text {th }}$ ). 


\subsubsection{Dynamic response of the ILMZ}

A modulated reverse voltage is applied to arm A of the modulator section (Fig. 21). The resulting modulated refractive index leads to the modulation of the light output of the laser which is biased above threshold. In the analysis, the small modulation of $\alpha_{\mathrm{A}}$ is taken into account in addition to the modulation of $n_{\mathrm{A}}$. The frequency chirp originates from two effects:

1) due to the optical feedback, the modulation of $r_{R}$ induces a change of the threshold carrier density of the laser and consequently a variation of the carrier density $N$. This leads to a modulation of $n_{1}$ and of the lasing wavelength.

2) Simultaneously, the modulation of $\alpha_{\mathrm{A}}$ and $n_{\mathrm{A}}$ induces a change of the phase of the light at the modulator output. This contributes to an additional frequency chirp [Marcuse 90].

In this model, we have neglected the spatial variation of the carrier distribution along the DFB laser section (spatial hole burning), the reflectivity at the laser/modulator interface (justified in the butt-coupling scheme [Simes 92]), the spontaneous emission coupled into the mode and the optical gain saturation in the laser section.

The small signal modulation is discussed in details in [Brosson 98b] The model gives analytical expressions of the complex AM and FM intrinsic responses $\delta P / \delta V$, $\delta f / \delta V$ and $\delta N / \delta V$ for $2 \times 1$ and $2 \times 2$ modulators. $\delta f / \delta V$ is the sum (complex) of three terms:

$(\delta f / \delta V)_{1}$, the direct modulation by the modulator, proportional to the modulation frequency $\Omega$.

$(\delta f / \delta V)_{2}$, related to optical feedback, constant with $\Omega$.

$(\delta f / \delta V)_{3}$, related to the indirect modulation of the carrier density in the laser section induced by optical feedback. The resonant FM response $(\delta f / \delta V)_{3}$ is proportional to the carrier density modulation $\delta N / \delta V$.

These contributions are shown in Fig. 22. The small signal analysis shows a strong increase or decrease of the FM response around the resonance frequency, depending on the facet phase.

The effective reflectance $\left|r_{\mathrm{R}}\right|$, the Henry parameter $\alpha_{\mathrm{H} 2}$ and the derivative of the normalized absorption $L_{2} \times \partial \alpha_{2} / \partial V$, have to be kept as low as possible in order to ensure a low frequency chirping behavior. A residual reflectivity lower than $10^{-4}$ is required (Fig. 23) to ensure low feedback induced frequency chirp. A lower chirp is obtained for the ILMZ compared to the ILM. Furthermore, at output B of the $2 \times 2$ ILMZ, as well as for the $\pi$-phase-shifted $2 \times 1$ ILMZ, the extinction ratio is higher and the effective chirp parameter is negative and small on the 0 to $V_{\pi}$ voltage range, while keeping a reasonably high output power. 


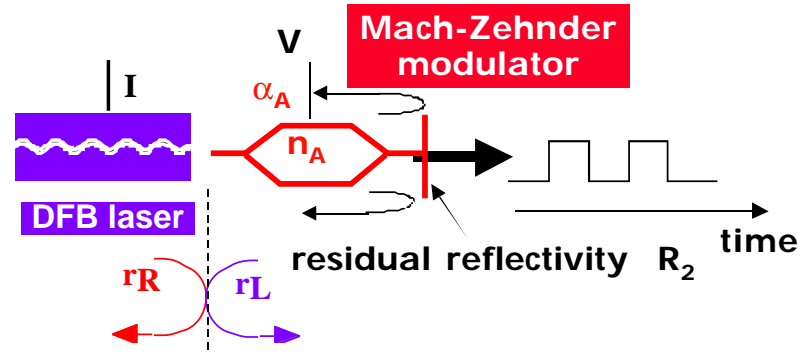

Figure 21. Origin of the feedback induced frequency chirp in ILMZ.
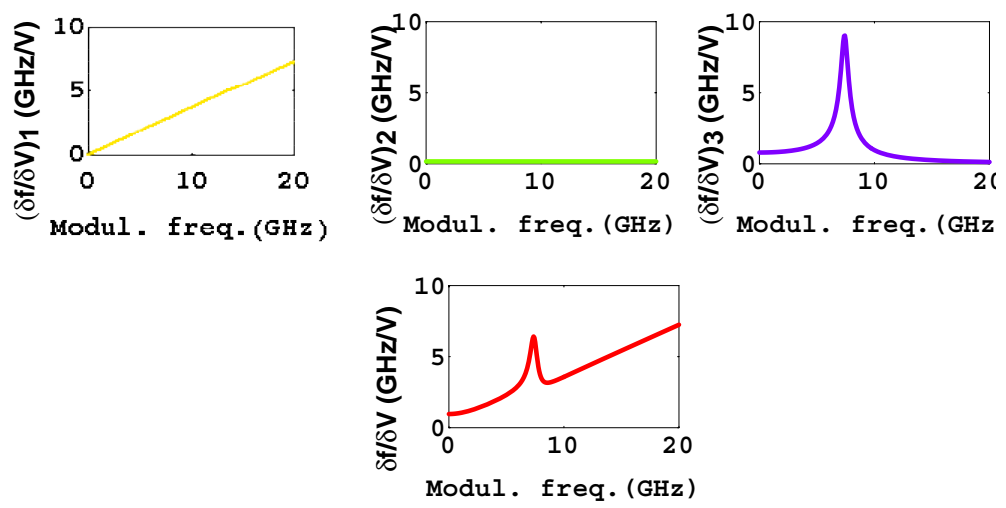

Figure 22. - FM response of the ILMZ. $\delta f / \delta V$ is the sum (complex) of three terms: $(\delta f / \delta V)_{1}$, the direct modulation by the modulator, proportional to the modulation frequency $\Omega ;(\delta f / \delta V)_{2}$, related to optical feedback, constant with $\Omega ;(\delta f / \delta V)_{3}$, resonant FM, related to the indirect modulation (induced by optical feedback) of the carrier density in the laser section.

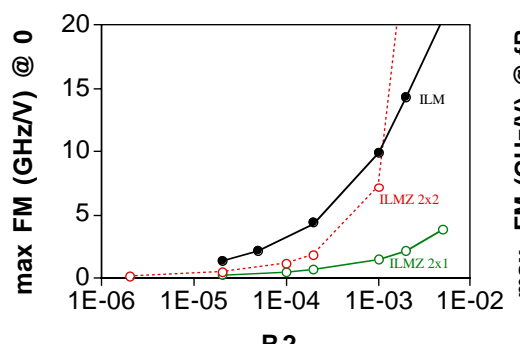

(a)

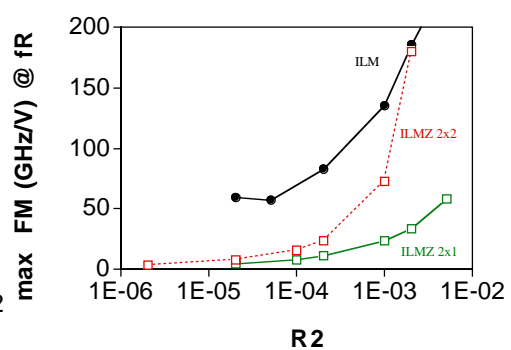

(b)

Figure 23. - Frequency modulation $(\delta f / \delta V)_{0} \operatorname{Max}$ and $(\delta f / \delta V)_{\mathrm{R} \text { Max }}$ for the worst phase $\varphi_{2}$ value, $-2 \mathrm{~V}$ bias, $\alpha_{\mathrm{H} 2}=13.5$ and comparison with the Integrated Laser electro-absorption Modulator (ILM) [Brosson 96] with $\alpha_{\mathrm{H} 2}=-1$. In the case of the $2 \times 2$ ILMZ, results are relative to the favorable (with negative effective chirp parameter) output B. (a) Frequency modulation $(\delta f / \delta V)_{0}$ Max at zero frequency. (b) Frequency modulation $(\delta f / \delta V)_{\mathrm{R} \text { Max }}$ at resonance. 
Based on this model, we have proposed a simple method to characterize the feedback induced chirp in an Integrated Laser Mach-Zehnder modulator by measuring the AM response at the back facet output of the laser [Brosson 98a]. Transmission at $2.5 \mathrm{Gbit} / \mathrm{s}$ over standard dispersive fiber $(1086 \mathrm{~km})$ using an ILMZ with a negative power penalty has been reported [Delansay 98]. The negative power penalty is due to pulse compression consequent to negatively chirped signals.

\section{SEMICONDUCTOR OPTICAL AMPLIFIER (SOA)}

\subsection{Introduction}

A Semiconductor Optical Amplifier (SOA) is obtained by Anti-Reflection (AR) coating its facets in order to prevent oscillation of the natural Fabry-Perot cavity. The original application of these devices was their use as in-line amplifiers in optical fiber links. Around 1990, in-line amplification was widely and successfully used with Erbium Doped Fiber Amplifiers (EDFA) due to their better performances (lower noise) compared to the intrinsic noise of SOAs (spontaneous emission) and compatibility with optical fibers. SOAs can be used as efficient and compact optical gates in photonic switching matrix.

Depending on the (AR) coating, two types of SOAs can be obtained: the resonant SOA with residual reflectivity around $R=10^{-2}$ and the Travelling Wave (TW) SOA with $R \leq 10^{-4}$. For the resonant SOA, the gain ripple is high (10-20 dB) and the bandwidth is very narrow $(2-10 \mathrm{GHz})$. For TW SOAs, the gain ripple is low ( $<$ a few $\mathrm{dBs}$ ) and the bandwidth is high $(3 \mathrm{THz})$. For optical applications in a switching matrix, the gain ripple must be as low as $0.3 \mathrm{~dB}$ and TW SOAs are required. SOAs can also be used as wavelength converters and all-optical lightwave 3- $\mathrm{R}$ regeneration [Chiaroni 97]. The 3-R regeneration means Re-amplification, Re-shaping and Re-timing. All-optical 3-R regeneration has been demonstrated using a wavelength converter (SOA under cross-gain modulation) associated with a second wavelength converter (2 SOAs in a Mach-Zehnder). This last application will not be discussed.

\subsection{Principle of operation}

Figure 24 shows an ideal TW SOA $(R=0)$. An input optical signal of intensity $I$ injected in the SOA waveguide is amplified according to

$$
I=I_{0} \exp \left(g_{\text {net }} L\right)
$$

where $g_{\text {net }}=\Gamma g-\alpha$ is the net gain, $\Gamma, g$, and $\alpha$ are respectively the optical confinement factor, the material gain and the optical loss.

The single pass gain is

$$
G_{\mathrm{s}}=\exp \left(g_{\text {net }} L\right)
$$




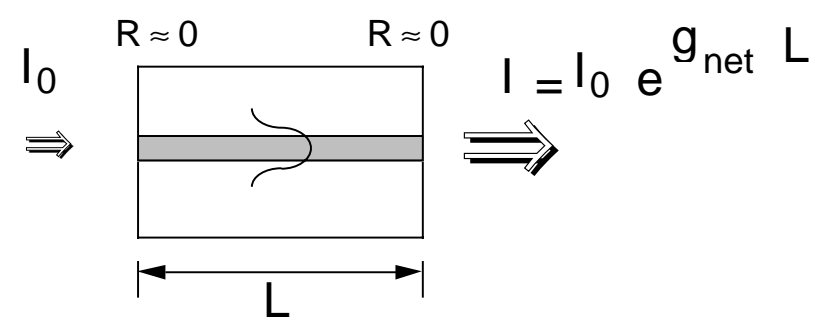

Figure 24. Principle of operation of the Semiconductor Optical Amplifier.

The gain of the SOA is often defined in $\mathrm{dB}$

$$
\begin{aligned}
G_{\mathrm{s}}(\mathrm{dB}) & =10 \log \left(G_{\mathrm{s}}\right) \\
& =10 \log (\mathrm{e}) g_{\text {net }} L \\
& =4.3 g_{\text {net }} L .
\end{aligned}
$$

With AR coating technology using $\mathrm{TiO}_{2} / \mathrm{SiO}_{2}$ layers, the lowest achievable reflectivity is $R=10^{-5}$. Tilted facets $\left(7^{\circ}\right)$ combined with AR coating allow a highly reproducible low effective residual reflectivity.

The gain of the amplifier is determined by the multiple reflections at the mirror facets of reflectivities $R_{1}$ and $R_{2}$. Within the rough approximation of a uniform carrier distribution along the $\mathrm{SOA}$, the small signal gain (unsaturated gain for $P_{\text {in }}<-30 \mathrm{dBm}$ ) is given by

$$
G(\lambda)=\frac{\left(1-R_{1}\right)\left(1-R_{2}\right) G_{\mathrm{s}}}{\left[1-\sqrt{R_{1} R_{2}} G_{\mathrm{s}}\right]^{2}+4 \sqrt{R_{1} R_{2}} G_{\mathrm{s}} \sin ^{2}\left[\left(k-k_{\mathrm{r}}\right) L\right]}
$$

where $k=2 \pi n_{\text {eff }} / \lambda, k_{\mathrm{r}}=2 \pi n_{\mathrm{eff}} / \lambda_{\mathrm{r}} ; \lambda$ is the wavelength of the input signal and $\lambda_{r}$ the wavelength of the resonant Fabry-Perot modes. Equation (11) neglects gain saturation by the Amplified Spontaneous Emission (ASE) and as Gs approaches the threshold value $1 / \sqrt{R_{1} R_{2}}$, gain ripple becomes infinite and the gain ripple given by (4.4) is over-estimated. Actually, both the ASE (determined by the spontaneous emission factor $\beta$ ) and the signal saturate the gain and this leads to a non uniform longitudinal carrier distribution [Brosson 94].

Gain saturation has the advantage of reducing the gain ripple. For $R_{1}=R_{2}=10^{-4}$, $P_{\text {in }}=-40 \mathrm{dBm}, \beta=10^{-3}$, taking into account gain saturation, the gain ripple is reduced below $2 \mathrm{dBm}$ (Fig. 25). For $\beta=4 \times 10^{-3}$, the gain ripple is reduced ( $<1 \mathrm{~dB}$ ), in agreement with experimental results.

The key parameters required for a SOA are

1) a low residual reflectivity $\left(R \leq 10^{-4}-10^{-5}\right)$ to ensure low gain ripple $(\leq 0.5 \mathrm{~dB})$;

2) low optical loss;

3) high material gain to ensure low drive current operation (20-30 dB fiber to fiber gain for $100 \mathrm{~mA}$ ); 


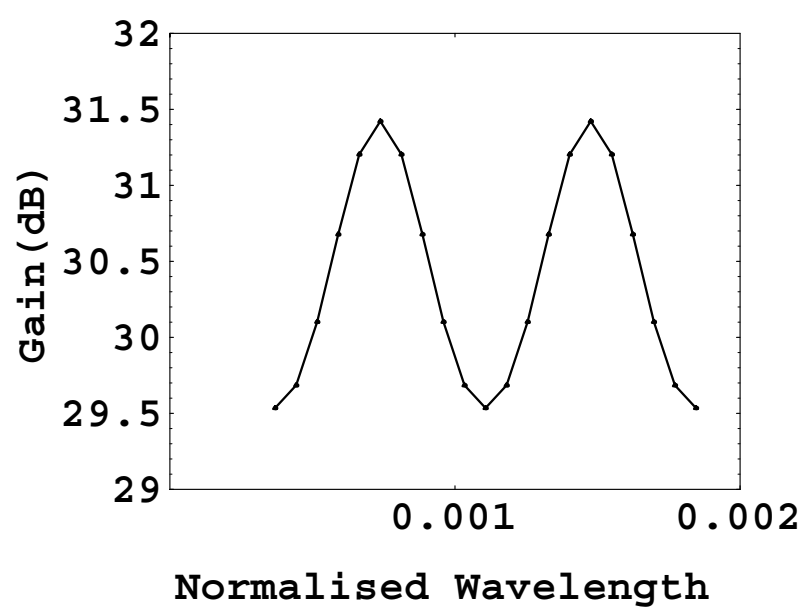

Figure 25. - Gain as a function of incident signal wavelength for $J / J_{\text {th }}=0.9, R_{1}=R_{2}=10^{-4}$, $P_{\text {in }}=-40 \mathrm{dBm}$ and a spontaneous emission factor $\beta=10^{-3}$.

4) low polarization sensitivity since the polarization of optical signal issued from a fiber is usually random. Since material gain is isotropic in bulk material, polarization sensitivity as low as $0.3 \mathrm{~dB}$ can be achieved with a bulk near square $(0.4 \mu \mathrm{m} \times 0.6 \mu \mathrm{m})$ active waveguide SOA with almost same confinement factor for both polarizations. The difficulty is to control the stripe width $<0.6 \mu \mathrm{m}$. In bulk SOAs, equalization of the modal gain (confinement factor, material gain product) is obtained using bulk-tensile strained SOAs (higher TM material gain) to compensate the lower TM optical confinement factor of rectangular waveguides;

5) High saturation power. The output saturation power $P_{\text {sat }}^{\text {out }}$ is defined as the output power for which the gain is reduced by $3 \mathrm{~dB}$. $P_{\text {sat }}^{\text {out }}$ is proportional to $1 / \Gamma$ and therefore improved (increased) in QW SOAs;

6) high coupling efficiency using integrated mode-expanding tapered waveguides at the output facets.

Compromises have to be accepted since all these requirements cannot be obtained simultaneously. With the use of Quantum Well SOAs, requirements 1-3 can be satisfied. Polarization sensitivity is intrinsic in quantum well SOAs $\left(G_{\mathrm{TE}}>G_{\mathrm{TM}}\right)$. It can be reduced by combining compressive strained QWs (higher TE gain ) and tensile strained QWs (higher TM gain). Requirement 5 is needed in WDM applications (the SOA can saturate by increasing the number of channels) since saturation of the SOA induces crosstalk between channels. A specific SOA device (Gain Clamped SOA) has been proposed and developed [Doussière 94b] to solve this problem. 

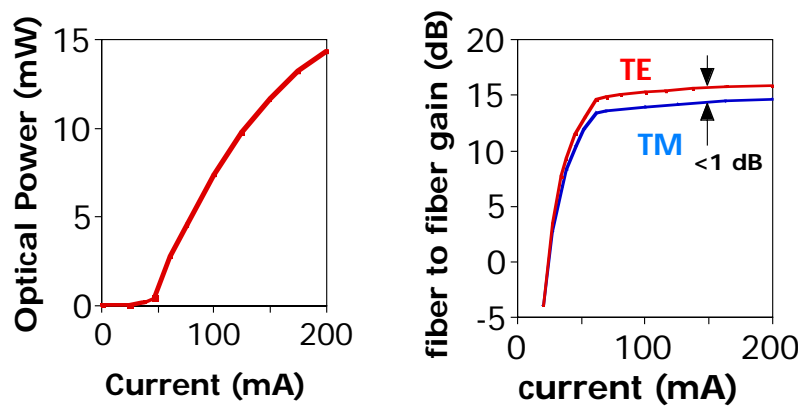

Figure 26. - Gain clamping by laser oscillation [Doussière 94b]. The device is lasing at wavelength $1580 \mathrm{~nm}$ and stabilized optical gain is available in the range $1550 \pm 20 \mathrm{~nm}$. Laser threshold is around $50 \mathrm{~mA}$. The gain saturates above $50 \mathrm{~mA}$.

\subsection{The Gain Clamped SOA}

\subsubsection{Why a Gain Clamped SOA?}

In optical switching systems, SOAs as optical gate have many advantages:

- compensation of coupling loss;

- high extinction ratio $(40 \mathrm{~dB})$;

- $G_{\mathrm{TE}} \approx G_{\mathrm{TM}}$ with suitable design.

However the gain saturates when increasing the number of wavelength channels (or the input power) in the same SOA. The saturation of the gain by one channel modifies the response of the other channels and produces crosstalk. The objective of the Gain Clamped SOA is to prevent gain saturation.

\subsubsection{Principle of operation of the Gain Clamped SOA}

The clamping [Doussière 94b] of the gain is obtained by forcing the SOA to lase at a wavelength $\lambda_{\text {laser }}$ lying outside the desired bandwidth of the SOA (for instance $\lambda_{\text {laser }}=1580 \mathrm{~nm}$ for $\lambda_{\text {signal }}=1550 \pm 20 \mathrm{~nm}$ ).

Due to lasing, the carrier density $N$ saturates and the optical gain which is approximately proportional to $N$ saturates too, whatever the input signal level. Of course, the gain is stabilized, but to a lower level compared to the standard SOA. The saturation output power turns out enhanced ( $+10 \mathrm{dBm}$ instead of $0 \mathrm{dBm})$.

Figure 26 shows the experimental light output characteristic (Amplified Spontaneous Emission, ASE) and the fiber-to-fiber gain of the GC SOA as a function of current. At the laser threshold $\left(I_{\text {th }} \approx 50 \mathrm{~mA}\right)$, the gain is clamped as expected. The polarization sensitivity of this bulk SOA is $<1 \mathrm{~dB}$.

Low fiber coupling loss is achieved through optical mode size shaping using a tapered waveguide at the end of the SOA (Fig. 27) in association with transverse evanescent coupling from the upper active SOA towards the passive waveguide. 


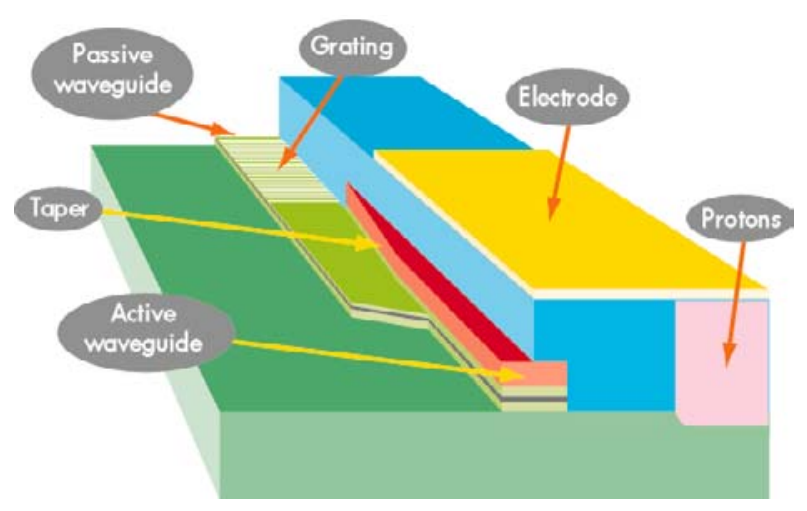

Figure 27. - Gain Clamped SOA with taper [Doussière 94b]. The coupling efficiency with fiber is improved with the integrated mode expanding tapered waveguides at the output facets. Lasing at $1580 \mathrm{~nm}$ is obtained thanks to the Bragg reflector (passive grating).

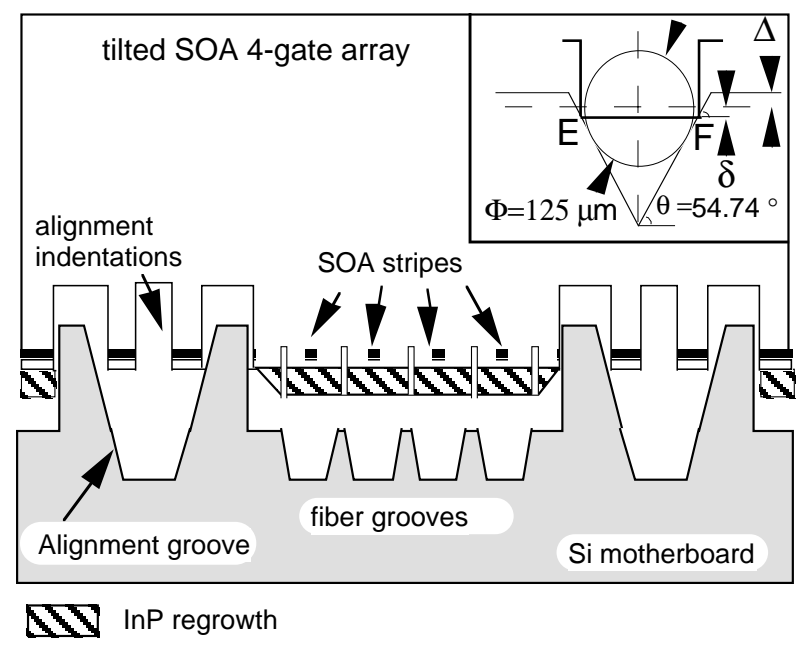

Figure 28. - Tilted SOA 4-gate array flip-chip mounted on a Si motherboard (not scaled). Insert: design of the alignment indentation, $E F=\Phi / \sin \theta-2 \delta / \operatorname{tg} \theta$ determines the vertical alignment.

\subsection{The 4-gate SOA array}

Due to their high gain, low driving current, low polarization sensitivity [Doussière 94a], high extinction ratio and fast switching capability [Fernier 92], SOAs have been proposed [Gabriagues 91] and used successfully as discrete optical gates in a $4 \times 4$ ATM optical switch block at $2.5 \mathrm{~Gb} / \mathrm{s}$ [Gabriagues 93, Chiaroni 93]. 


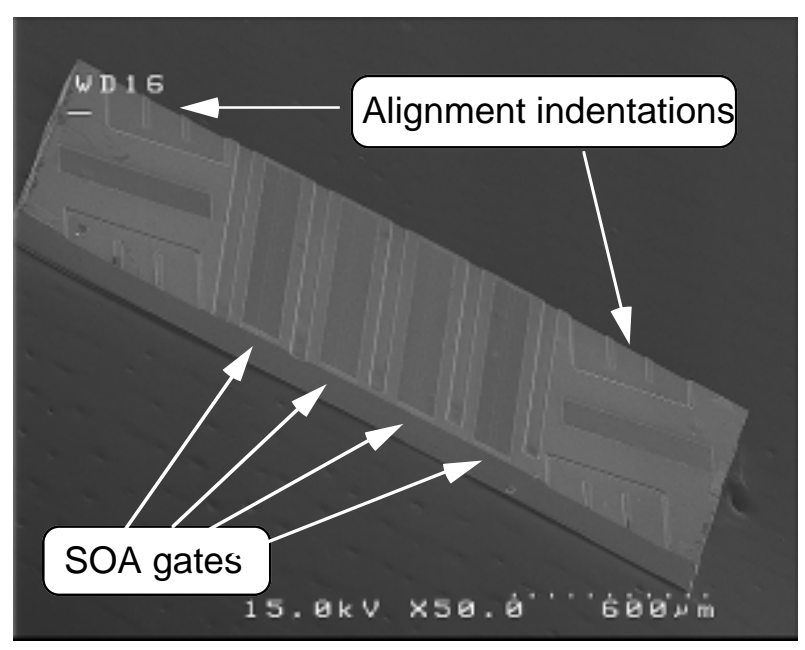

Figure 29. SEM viewgraph of the tilted $\left(7^{\circ}\right)$ SOA 4-gate array chip.

A large size optical switching matrix is possible only with integrated SOA gates due to the large number of gates required [Renaud 96]. Reducing the number of discrete components lowers the packaging coast of the switching matrix since dynamic alignment is not required.

A 4-gate $1.55 \mu \mathrm{m}$ SOA (Fig. 28) flip-chip soldered on Si motherboard has been demonstrated [Hunziker 95], [Leclerc 95]. Alignment indentations are defined on the SOA array and fit into V-grooves defined in the Si motherboard and the fibers fit in separate V-grooves as shown in Figure 28, providing automatic alignment of the 4 gates and 8 fibers in the vertical and lateral directions.

A SEM view of the tilted SOA 4-gate array is shown in Figure 29. The dimensions of the array are $2300 \mu \mathrm{m}$ wide and $800 \pm 5 \mu \mathrm{m}$ long, the 4 SOA stripes being spaced by $250 \mu \mathrm{m}$.

Measurements of the fiber-to-fiber gain on the packaged module are shown in Figure 30. The gain characteristics are uniform among the 4 gates, with a fiber-to-fiber gain of $14 \mathrm{~dB}$ and $1.3 \mathrm{~dB}$ polarization sensitivity. Penalty-free switching experiment at $2.5 \mathrm{~Gb} / \mathrm{s}$ (Asynchronous Transfer Mode Optical Switching, ATMOS, Fig. 31) using this 4-gate SOA array has been demonstrated [Gavignet 95] with switching times shorter than 400 ps. A $4 \times 4$ ATM switch block operating at $2.5 \mathrm{~Gb} / \mathrm{s}$ is shown in Figure 32.

Wavelength is used to route the cells towards the required address (routing tag). To avoid cell contention, the cells are directed to fiber delay lines of $0,1,2,3,4 \mathrm{~T}$, using optical gates. T is the ATM cell duration $(\approx 200 \mathrm{~ns}$ corresponding to $40 \mathrm{~m}$ optical fiber).

New generations of 8-gate Gain Clamped SOAs [Dorgeuille 2000] have been recently developed, demonstrating WDM switching experiments (16 wavelengths $\times$ $10 \mathrm{~Gb} / \mathrm{s})$. 
Gain versus input power. Array 9147A1 10. Input wavelength $=1553 \mathrm{~nm}$. I = $80 \mathrm{~mA}$. $\mathrm{T}=25^{\circ} \mathrm{C}$.

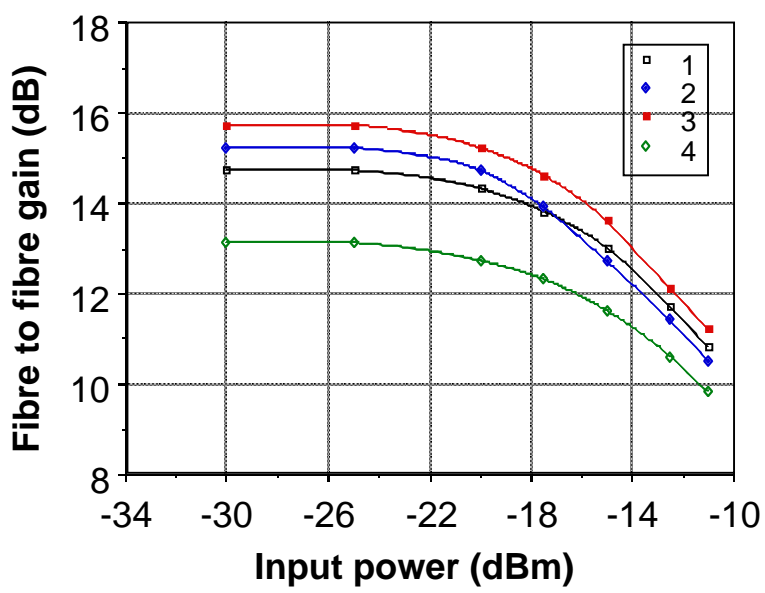

Figure 30. Fiber-to-fiber gain for the 4 SOAs of the gate-array module.

Asynchronous Transfer Mode (ATM)

\begin{tabular}{|l|l|}
\hline \multicolumn{2}{|c|}{53 bytes } \\
\hline $\begin{array}{l}5 \text { bytes } \\
\text { header }\end{array}$ & $\begin{array}{l}48 \text { bytes } \\
\text { payload }\end{array}$ \\
\hline
\end{tabular}

ATM Optical Switching (ATMOS)

64 bytes

\begin{tabular}{|c|c|c|c|}
\hline $\begin{array}{c}3 \text { bytes } \\
\text { guard }\end{array}$ & \begin{tabular}{|l|}
8 bytes \\
routing
\end{tabular} & $\begin{array}{l}5 \text { bytes } \\
\text { header }\end{array}$ & $\begin{array}{l}48 \text { bytes } \\
\text { payload }\end{array}$ \\
\hline $9.6 \mathrm{~ns}$ & $25.7 \mathrm{~ns}$ & $16.1 \mathrm{~ns}$ & $154.3 \mathrm{~ns}$ \\
\hline
\end{tabular}

Figure 31. ATM Optical Switching cell. 


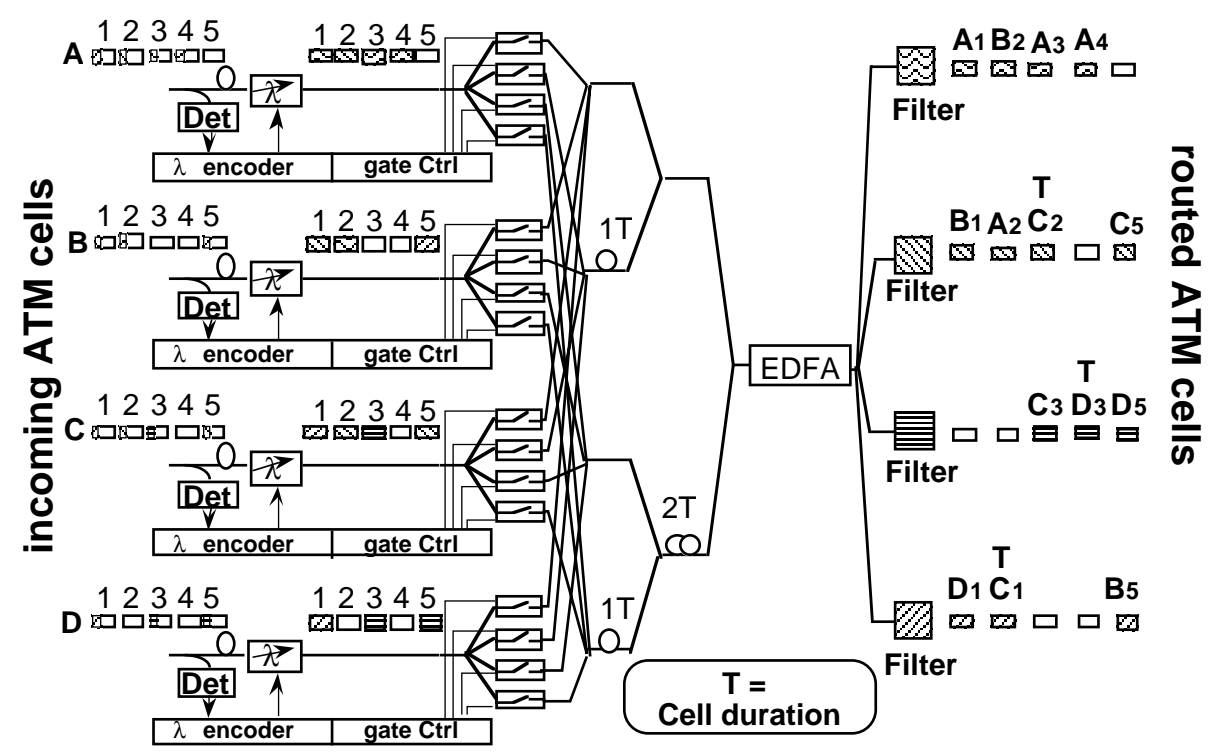

Figure 32. $-4 \times 4$ ATM block for $2.5 \mathrm{~Gb} / \mathrm{s}$ switching experiments (ATMOS RACE project) with of 4 4-gate arrays (16 optical gates). The incoming ATM cells are routed by opening the SOA gates. Contention of the cell is avoided thanks to the optical fiber delay lines (1T, 2T, 3T). 


\section{WAVELENGHT CONVERTER}

\subsection{Introduction}

Wavelength converters are key components for Wavelength Division Multiplexing (WDM) photonic switching systems [Gabriagues 91] and switching matrix using wavelength routing. In the 1530-1560 nm available bandwidth of the Erbium Doped Fiber Amplifier (EDFA), the number of wavelengths channels is limited to $\approx 40$ for a $100 \mathrm{GHz}$ span and wavelengths must be reused. In other words, wavelength conversion is required. We will not discuss opto-electronic conversion and will focus on all-optical conversion which can be obtained with bi-stable tunable lasers or SOAs. For system applications, the device must be able to convert the signal to the same wavelength.

\subsection{All-optical wavelengths converters}

The objective of these converters is to obtain at wavelength $\lambda_{\text {out }}$ a replica of the $\lambda_{\text {in }}$ optical input signal and to avoid optic-electric conversion. In most of these devices, the output wavelength is tuned by adjusting the electric current through a passive DBR section. The increase of the carrier concentration in the DBR section induces a reduction of the refractive index and consequently a decrease of the Bragg wavelength. Depending on the mechanism, the extinction ratio can be improved or degraded.

\subsubsection{Bistable Distributed Bragg Reflector (DBR) laser}

The device (Fig. 33) consists of an active gain section, a Bragg section and a saturable absorber section. In the saturable absorber section (10-20 $\mu \mathrm{m}$ long), the active layer has been proton implanted to ensure bistable operation. The tunable Bragg section allows wavelength tuning (by adjusting $I_{\text {Bragg }}$ ) of the laser at the required output wavelength $\lambda_{\text {out }}$.

The laser is biased slightly below threshold and the optical input signal triggers the DBR laser. The wavelength range of this device is limited by the condition $\lambda_{\text {in }}<\lambda_{\text {out }}$ since optical pumping energy must be higher than the signal output energy. The maximum bit rate is determined by the effective carrier lifetime and $1 \mathrm{~Gb} / \mathrm{s}$ has been reported [Duan 93, Serre 93]. Table I gives the main features of this device.

\subsubsection{Carrier depletion in the gain section of a Distributed Bragg Reflector (DBR) laser}

The device (Fig. 34) consists of an active gain section, a passive Bragg section and a passive phase section [Mikkelsen 93a, Ottolenghi 93]. The laser is biased slightly above threshold. The optical input signal $\left(\lambda_{\text {in }}\right)$ is amplified in the gain section and this contributes to deplete the gain (eventually below threshold) leading to a reduction of the power of the DBR emitting at $\lambda_{\text {out }}$. An inverted replica of the input signal is obtained at $\lambda_{\text {out }}$. 


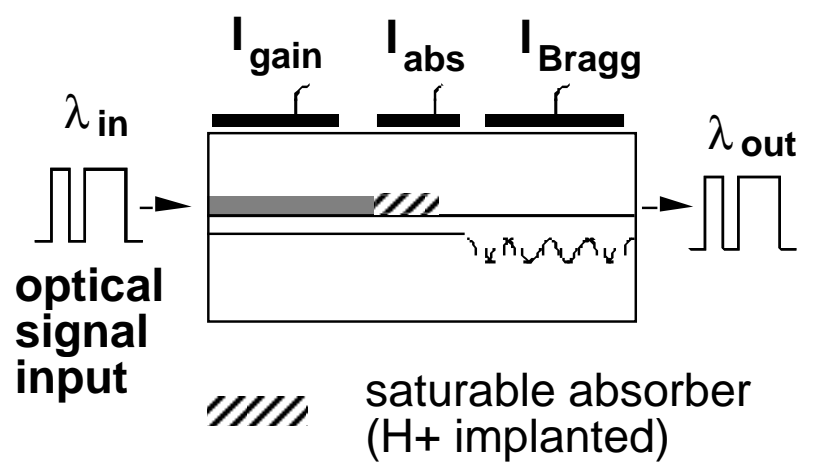

Figure 33. All-optical wavelength converter using a bistable DBR laser.

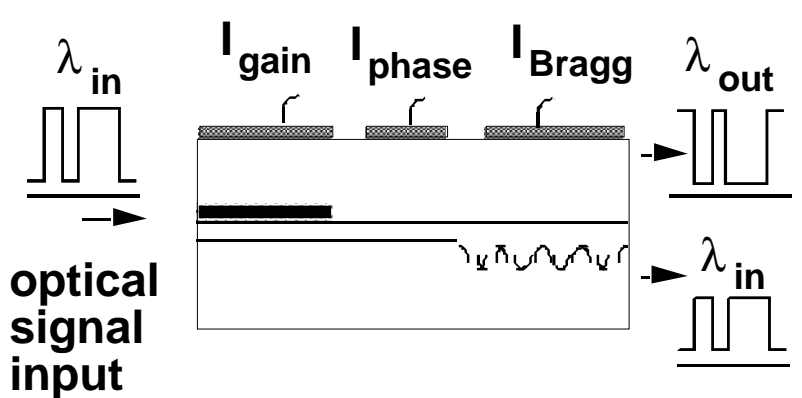

Figure 34. - All-optical wavelength converter using carrier depletion in the gain section of a DBR laser.
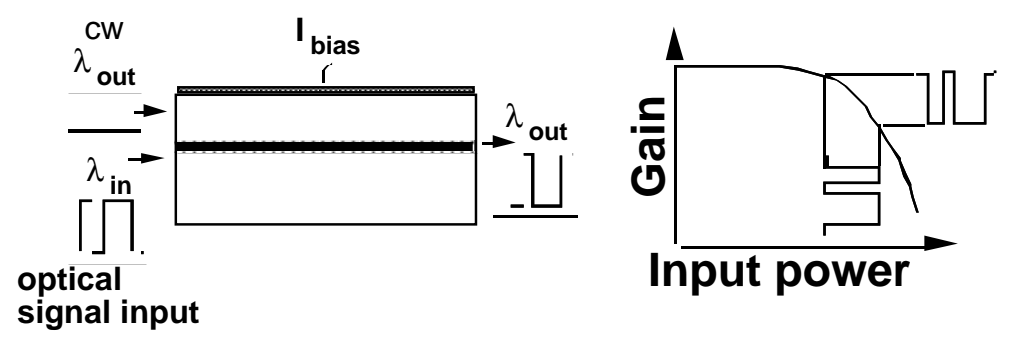

Figure 35. - All-optical wavelength converter using gain saturation in a Semiconductor Optical Amplifier. 
Table I. - Wavelength converter using a bi-stable tunable Distributed Bragg Reflector (DBR) laser. The condition $\lambda_{\text {in }}<\lambda_{\text {out }}$ is required to allow optical pumping.

\begin{tabular}{|l|l|}
\hline Bit rate $(\mathrm{Gb} / \mathrm{s})$ & 1 \\
\hline$\lambda_{\text {in }}$ range $(\mathrm{nm})\left(\right.$ with $\left.\lambda_{\text {in }}<\lambda_{\text {out }}\right)$ & 20 \\
\hline$\lambda_{\text {out }}$ tunability $(\mathrm{nm})$ & 3 \\
\hline Extinction ratio regeneration & yes \\
\hline Polarization sensitivity input/output & high/low \\
\hline
\end{tabular}

Table II. - Wavelength converter using Cross-Gain Modulation (XGM) due to gain saturation in the gain section of a tunable Distributed Bragg Reflector (DBR) laser. Same wavelength conversion $\lambda_{\text {in }}=\lambda_{\text {out }}$ is not possible.

\begin{tabular}{|l|l|}
\hline Bit rate $(\mathrm{Gb} / \mathrm{s})$ & 5 \\
\hline$\lambda_{\text {in }}$ range $(\mathrm{nm})\left(\right.$ with $\left.\lambda_{\text {in }} \neq \lambda_{\text {out }}\right)$ & 40 \\
\hline$\lambda_{\text {out }}$ tunability $(\mathrm{nm})$ & 3 \\
\hline Extinction ratio regeneration & yes \\
\hline Polarization sensitivity input/output & high/high \\
\hline
\end{tabular}

Table III. - Wavelength converter using Cross-Gain Modulation (XGM) due to gain saturation in a SOA. Same wavelength conversion $\lambda_{\text {in }}=\lambda_{\text {out }}$ is not possible.

\begin{tabular}{|l|l|}
\hline Bit rate $(\mathrm{Gb} / \mathrm{s})$ & $\begin{array}{l}2.5 \\
20 @ \text { @igh power }\end{array}$ \\
\hline$\lambda_{\text {in }}$ range $(\mathrm{nm})\left(\right.$ with $\left.\lambda_{\text {in }} \neq \lambda_{\text {out }}\right)$ & 40 \\
\hline$\lambda_{\text {out }}$ tunability $(\mathrm{nm})$ & $\begin{array}{l}\text { Tunability of the } \mathrm{cw} \\
\text { laser }\end{array}$ \\
\hline Extinction ratio regeneration & no \\
\hline Polarization sensitivity input/output & low/low \\
\hline chirp & degraded \\
\hline
\end{tabular}


The tunable Bragg section allows wavelength tuning (by adjusting $I_{\text {Bragg }}$ ) of the laser at the required output wavelength $\lambda_{\text {out }}$. The wavelength range of this device is limited by the condition $\lambda_{\text {in }} \neq \lambda_{\text {out }}$. Furthermore, an output filter at $\lambda_{\text {out }}$ is required to reject the modulated input signal $\left(\lambda_{\text {in }}\right)$. Table II gives the main features of this device.

\subsubsection{Gain saturation in a Semiconductor Optical Amplifier (SOA)}

The device (Fig. 35) is a Semiconductor Optical Amplifier (SOA). The wavelength conversion mechanism is similar to the previous case. CW light (at the required wavelength $\lambda_{\text {out }}$ ) is injected into the SOA and amplified, resulting in a CW output beam in the absence of input signal. The optical signal (wavelength $\lambda_{\text {in }}$ ) and CW light (at the required wavelength $\lambda_{\text {out }}$ ) are injected into the SOA [Joergensen 92, Mikkelsen 93b]. The optical input signal is amplified in the SOA and this contributes to deplete the gain of the SOA leading to a reduction of the power of the output beam. An inverted replica of the input signal is obtained at $\lambda_{\text {out }}$. This technique is referred as Cross-Gain Modulation (XGM). Extinction ratio is degraded as well as chirp in the output signal.

A filter at $\lambda_{\text {out }}$ is required to reject the modulated input signal $\left(\lambda_{\text {in }}\right)$ and the Amplified Spontaneous Emission (ASE). Wavelength tuning is obtained using a CW tunable or standard DFB laser emitting at the required wavelength $\lambda_{\text {in }}$. The maximum bit rate is determined by the effective carrier lifetime and is improved by increasing the length of the SOAs (enhanced interaction length) and the photon density (smaller optical mode cross section) in the Mach-Zehnder. The low polarization sensitivity is obtained using bulk-tensile strain SOAs. Table III gives the main features of this device.

\subsubsection{Semiconductor Optical Amplifier (SOA) in a Mach-Zehnder interferometer}

Principle of operation and first experimental demonstrations

The device consists of a Semiconductor Optical Amplifier (SOA) in arm 1 of an optical fiber Mach-Zehnder interferometer [Durhuus 93, Mikkelsen 93b, Doussière 92a] (Fig. 36). The wavelength conversion mechanism is similar to the previous case but relies on refractive index change associated with gain saturation in the SOA.

CW light (at the required wavelength $\lambda_{\text {out }}$ ) is injected into the two arms of the Mach-Zehnder, amplified by the SOA and recombined, resulting in a CW output beam in the absence of input signal. The optical signal (wavelength $\lambda_{\text {in }}$ ) and CW light (at the required wavelength $\lambda_{\text {out }}$ ) are injected into the Mach-Zehnder. The optical input signal is amplified in the SOA and this contributes to deplete the gain and the carrier density in the SOA, inducing a refractive index change and consequently a phase difference $\Delta \varphi$ between the two arms of the Mach-Zehnder interferometer. $\Delta \varphi$ can be adjusted to $\Delta \varphi=\pi$ by changing the gain of the SOA, in order to obtain destructive interferences (out-of-phase conversion). An inverted replica of the input signal is obtained at $\lambda_{\text {out }}$. Adjusting $\Delta \varphi=0$ (in-phase conversion), a replica of the 


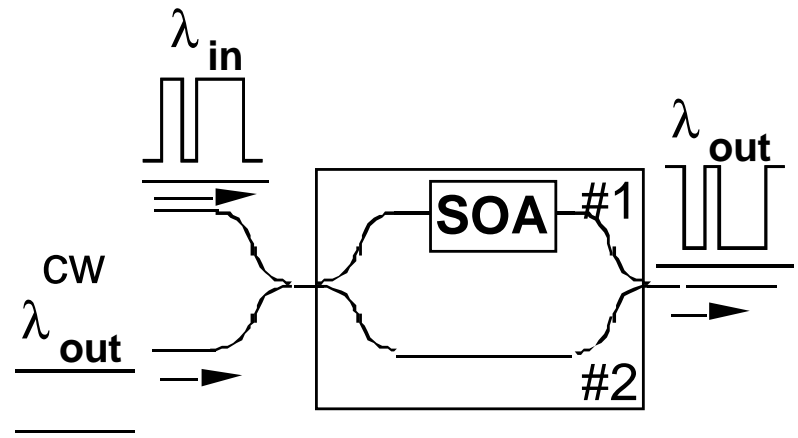

Figure 36. - All-optical wavelength converter using a Semiconductor Optical Amplifier in a Mach-Zehnder fiber interferometer, co-propagation configuration, out-of-phase conversion. An optical filter is necessary to reject the input signal $\left(\lambda_{\text {in }}\right)$. Consequently, same wavelength conversion $\lambda_{\text {in }}=\lambda_{\text {out }}$ is not possible.

input signal is obtained at $\lambda_{\text {out }}$. This technique is referred as Cross-Phase Modulation (XPM) since the phase modulation is translated into amplitude modulation of the CW beam.

A filter at $\lambda_{\text {out }}$ is required to reject the modulated input signal $\left(\lambda_{\text {in }}\right)$ for this copropagation configuration, and conversion to the same wavelength is not possible. In the counter-propagation configuration (the $\mathrm{CW}$ light beam is injected from the opposite side of the input signal), the input signal is rejected, the filter is not required and same wavelength conversion becomes possible. However, the maximum bit rate is lower for this configuration (due to the short interaction time between the beams, the mechanism is less effective). Wavelength tuning is obtained using a CW tunable or standard DFB laser emitting at the required wavelength $\lambda_{\text {in }}$.

The main advantage of this converter is the improvement of the extinction ratio. Furthermore since the gain modulation of the SOA is small compared to the XGM case, the converted output signal is nearly chirp-free. The sign of the residual chirp can be adjusted positive (in-phase conversion) or negative (out-of-phase conversion). This converter has been realized by integration of semiconductor passive waveguides and active SOAs in both or one arm of the Mach-Zehnder.

Table IV gives the main features of this device (co-propagation configuration).

Integrated all-active Mach-Zehnder wavelength converter, co-propagation

configuration

Instead of integrating passive waveguides and couplers with active SOAs in both or one arm of the Mach-Zehnder, all-active integration greatly simplifies the fabrication process and eliminates parasitic internal reflections [Janz 99a, Janz 99c, Dagens 99]. Coupling losses are compensated by the SOAs. Figure 37 shows such an integrated all-active wavelength converter.

The two SOAs in the arms of the Mach-Zehnder are long $(1.2 \mathrm{~mm})$ to allow saturation of the gain and enhance cross phase modulation of the $\mathrm{CW}$ beam by the 


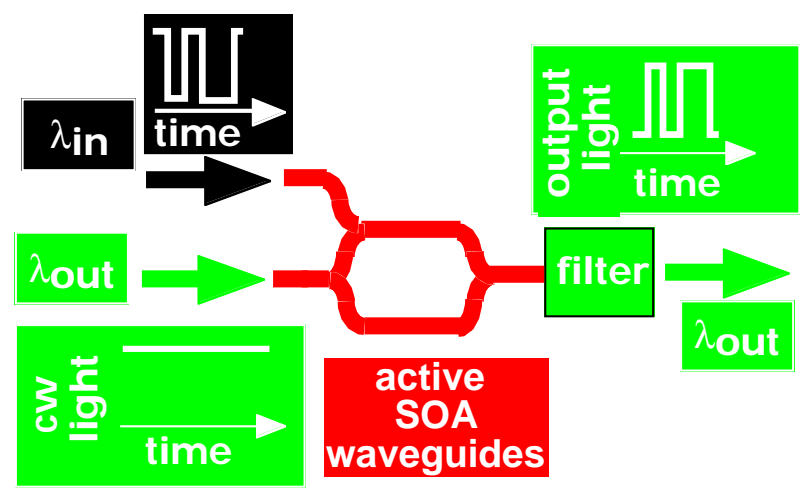

Figure 37. - Integrated all-active Mach-Zehnder (MZ) wavelength converter, co-propagation configuration, out-of-phase conversion. The CW input, signal input, output and MZ waveguides are SOAs. Same wavelength conversion $\lambda_{\text {in }}=\lambda_{\text {out }}$ is not possible.

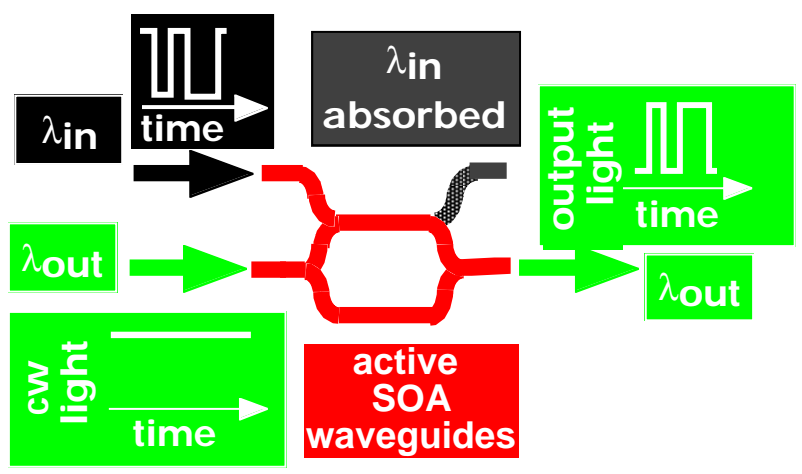

Figure 38. - Integrated all-active Dual-Order Mode (DOMO) Mach-Zehnder wavelength converter, co-propagation configuration, out-of-phase conversion. The arms of the MachZehnder are made of active SOA waveguides sustaining two lateral modes. Multi-Mode Interference (MMI) couplers are used to couple the input signal and the CW beam and to reject the input signal. Same wavelength conversion $\lambda_{\text {in }}=\lambda_{\text {out }}$ is possible.

signal. Co-propagation configuration (the $\mathrm{CW}$ light beam and input signal propagate in the same direction), allows high bit rates but requires filtering of the input signal and prohibits conversion to the same wavelength. The three SOAs respectively at the input signal, CW, and output signal ports are made as short as possible to avoid gain saturation. They provide compensation of the coupling losses and eventually can boost the input signal. Integrated mode-expanding tapers are included at the cleaved facets to improve coupling with the fibers. Wavelength conversion has been demonstrated at $10 \mathrm{~Gb} / \mathrm{s}$ with an input signal power of $-10 \mathrm{dBm}(10 \mathrm{~dB}$ improvement compared to the passive-active scheme)with a signal power dynamic range of $15 \mathrm{~dB}$. 
Table IV. - Wavelength converter using Cross-Phase Modulation (XPM) due to gain saturation in a SOA Mach-Zehnder interferometer. Same wavelength conversion $\lambda_{\text {in }}=\lambda_{\text {out }}$ is not possible.

\begin{tabular}{|l|l|}
\hline Bit rate $(\mathrm{Gb} / \mathrm{s})$ & $\begin{array}{l}2.5 \\
20\end{array}$ \\
\hline$\lambda_{\text {in }}$ range $(\mathrm{nm})\left(\right.$ with $\left.\lambda_{\text {in }} \neq \lambda_{\text {out }}\right)$ & 40 \\
\hline$\lambda_{\text {out }}$ tunability $(\mathrm{nm})$ & $\begin{array}{l}\text { Tunability } \\
\text { of the cw } \\
\text { laser }\end{array}$ \\
\hline Extinction ratio regeneration & yes \\
\hline Polarization sensitivity input/output & low/low \\
\hline chirp & $\begin{array}{l}\text { nearly } \\
\text { chirp-free }\end{array}$ \\
\hline
\end{tabular}

Table V. — Integrated all-optical, all-active Mach-Zehnder wavelength converters. Best performances (high bit rate and same wavelength conversion possible) are obtained with the Dual-Order Mode wavelength converter (DOMO).

\begin{tabular}{|c|c|c|c|}
\hline & \multicolumn{2}{|c|}{ co-propagation configuration } & Counter propagation \\
\hline & $\begin{array}{l}\mathrm{MZ} \lambda \lambda \text { converter } \\
L=1.2 \mathrm{~mm}\end{array}$ & $\begin{array}{l}\text { DOMO MZ } \lambda \text { converter } \\
L=1.8 \mathrm{~mm}\end{array}$ & $\begin{array}{l}\text { MZ } \lambda \text { converter } \\
L=1.2 \mathrm{~mm}\end{array}$ \\
\hline reference & [Janz 99a] & [Janz 99b] & [Janz 99a] \\
\hline Input signal rejection & \begin{tabular}{|l|} 
Filter required \\
$\lambda_{\text {in }} \rightarrow \lambda_{\text {in }}$ prohibited
\end{tabular} & $\begin{array}{l}28 \mathrm{~dB} \\
\lambda_{\text {in }} \rightarrow \lambda_{\text {in }} \text { possible }\end{array}$ & $\begin{array}{l}\text { Intrinsic } \\
\lambda_{\text {in }} \rightarrow \lambda_{\text {in }} \text { possible }\end{array}$ \\
\hline Input signal sensitivity & $-10 \mathrm{dBm}$ & & $-3 \mathrm{dBm}$ \\
\hline Input dynamic range & $15 \mathrm{~dB}$ & & $?$ \\
\hline $\begin{array}{l}\text { bit rate (experim.) } \\
\text { (expected) }\end{array}$ & $\begin{array}{l}10 \mathrm{~Gb} / \mathrm{s} \\
>10 \mathrm{~Gb} / \mathrm{s}\end{array}$ & $\begin{array}{l}10 \mathrm{~Gb} / \mathrm{s} \\
>10 \mathrm{~Gb} / \mathrm{s}\end{array}$ & $10 \mathrm{~Gb} / \mathrm{s}$ \\
\hline SNR & & $45 \mathrm{~dB}$ & \\
\hline $\begin{array}{l}\text { Extinction } \\
\text { ratio } \\
\text { regeneration }\end{array}$ & yes & yes & yes \\
\hline $\begin{array}{l}\text { Chirp } \\
>0 \text { if in-phase } \\
\text { conversion } \\
<0 \text { if out-phase } \\
\text { conversion }\end{array}$ & nearly chirp-free & nearly chirp-free & low \\
\hline
\end{tabular}


Integrated all-active Dual-Order Mode (DOMO) Mach- Zehnder wavelength converter, co-propagation configuration

This scheme [Janz 99b] allows both high conversion speed (taking advantage the high conversion speed of the co- propagation configuration) and conversion to the same wavelength (thanks to strong rejection of the input signal).

The arms of the Mach-Zehnder are made of active SOA waveguides $(1.8 \mathrm{~mm})$ sustaining two lateral modes. Multi-Mode Interference (MMI) couplers are used to couple the input signal and the $\mathrm{CW}$ beam and to reject the input signal as shown in Figure 38. Same design rules (short peripheral active guides, small optical mode cross section, bulk-tensile strained SOAs, integrated mode-expanding tapers at the cleaved facets) are used. Wavelength conversion at $10 \mathrm{~Gb} / \mathrm{s}$ with $28 \mathrm{~dB}$ rejection of the input signal have been demonstrated. Table V summarizes and compares the performances of these all-optical, all-active Mach-Zehnder wavelength converters.

\section{WAVELENGTH DEMULTIPLEXER (WDM)}

\subsection{Introduction}

In Wavelength Division Multiplexing systems (WDM), in order to increase the total bit rate capacity of a link without increasing the basic bit rate of the transmitter, several different signals at different wavelengths (channels) are transported simultaneously over the same optical fiber (Fig. 39). As an example the transmission over $6150 \mathrm{~km}$ of 32 channels modulated at $10 \mathrm{~Gb} / \mathrm{s}$ has been demonstrated [Gautheron 99b]. In order to extract (Fig. 40) one or several channels, Wavelength DeMultiplexers (WDMs) with the $100 \mathrm{GHz}$ ITU channel spacing $(0.8 \mathrm{~nm})$ are required. WDMs with closer channel spacing (50 GHz ITU grid) are required for Dense WDM. Such Wavelength DeMultiplexers are key components for WDM systems. The Wavelength demultiplexer is also referred as phase array.

\subsection{Wavelength Demultiplexer operating principle}

Figure 41a shows the example of 9 mutiplexed signals carried by 3 different wavelengths $\lambda_{1}, \lambda_{2}$ and $\lambda_{3}$. The purpose is to route these 9 wavelength-multiplexed signals towards their respective wavelength channel. The demultiplexer consists of 3 input and 3 output waveguides and an array of a large number of waveguides. The waveguide array is a kind of "super" Mach-Zehnder interferometer with many arms. Each arm has a different length (increment $\Delta L$ ). For waves travelling from the input waveguides towards port $0_{1}$, constructive interferences occur at $\lambda_{1}$. The same argument can be repeated for the remaining $\lambda_{2}$ and $\lambda_{3}$. A schematic representation of a 4-channel Wavelength Demultiplexer is shown in Figure 41b. The actual number of waveguides is 12 . Figure 42 shows the ridge waveguide structure. Single mode operation is obtained for a $2 \mu \mathrm{m}$ width and a $1.1 \mu \mathrm{m}$ thickness. The characteristics of the WDM are given in Table VI (see end of section). 


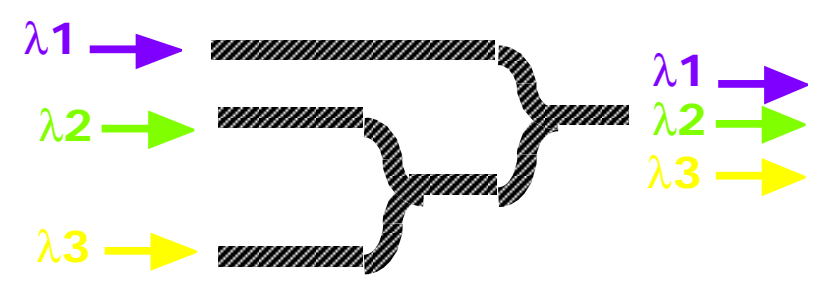

Figure 39. Wavelength multiplexing.

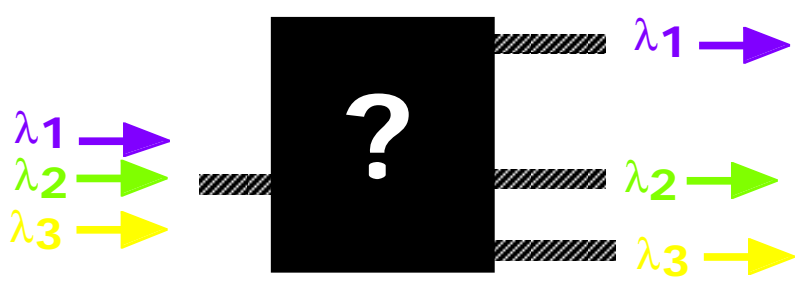

Figure 40. Wavelength demultiplexer.

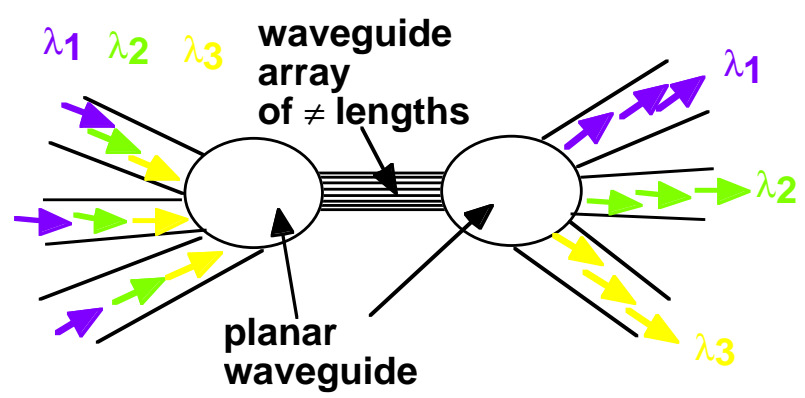

(a)

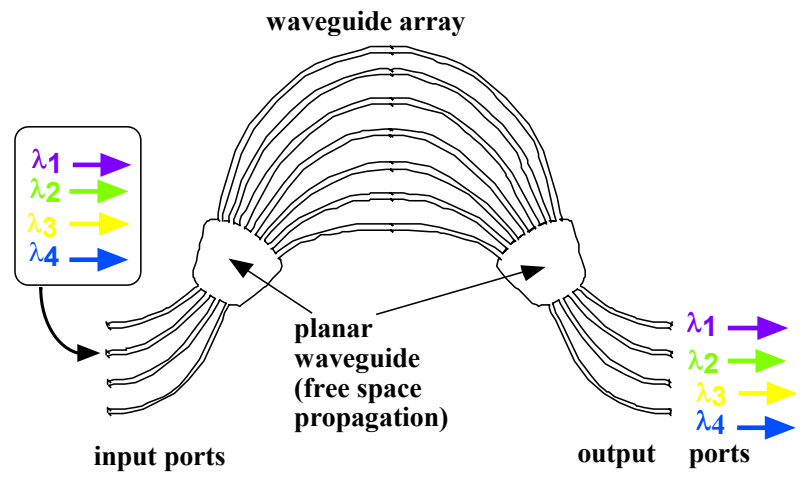

(b)

Figure 41. Wavelength demultiplexer, operating principle. 


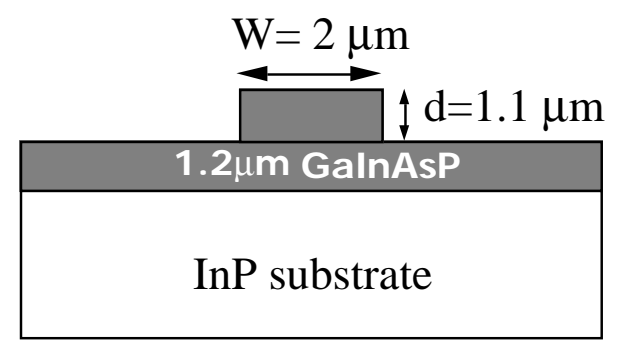

Figure 42. Ridge waveguide structure [Bissessur 95a].

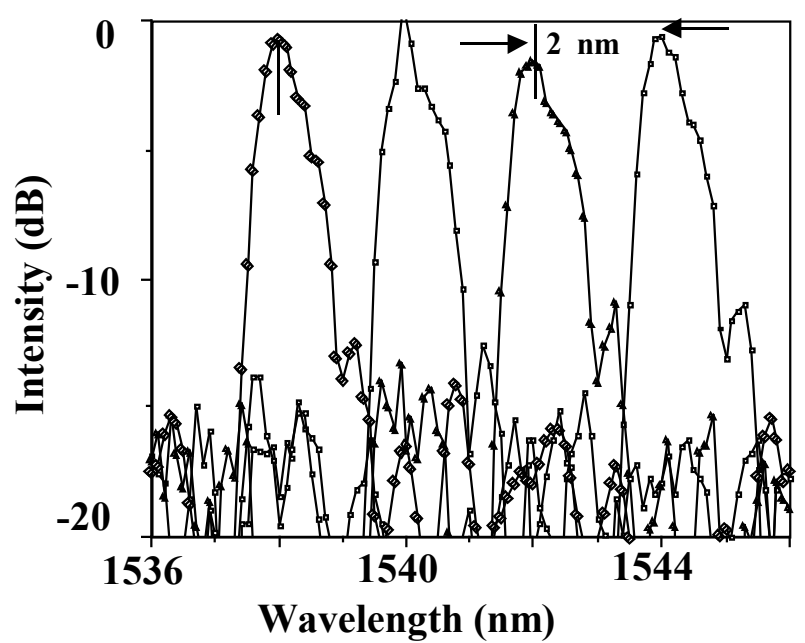

Figure 43. - Measured spectral response of the 4-channel Wavelength Demultiplexer. Light is coupled into one input guide and the output spectrum is measured for each of the 4 output waveguides. The 4 spectra are shown overlaid [Bissessur 95a].

Figure 43 shows the measured spectral response of the 4-channel WDM. Light is coupled into one input guide and the output spectrum is measured for each of the 4 output waveguides. The 4 spectra are shown overlaid.

Since the waveguides (Fig. 42) are not square, the effective index is different for the two polarizations and the maximum of the spectral response is polarization (Fig. 44) dependent $\left(\lambda_{\mathrm{TE}} \neq \lambda_{\mathrm{TM}}\right)$. However, it is possible to optimize the geometry $(w=2 \mu \mathrm{m})$ of the waveguide to obtain nearly zero [Bissessur 95b] birefringence $\left(\lambda_{\mathrm{TE}} \neq \lambda_{\mathrm{TM}}\right)$ as shown in Figure 44.

Figure 45 shows the measured spectral response of an extremely small 16-channel WDM. Its characteristics are given in Table VII. The record small size $\left(1.1 \times 1.1 \mathrm{~mm}^{2}\right)$ is obtained using small $(100 \mu \mathrm{m})$ radius curvature. This Wavelength Demultiplexer will be part of the wavelength selector (see next chapter). 


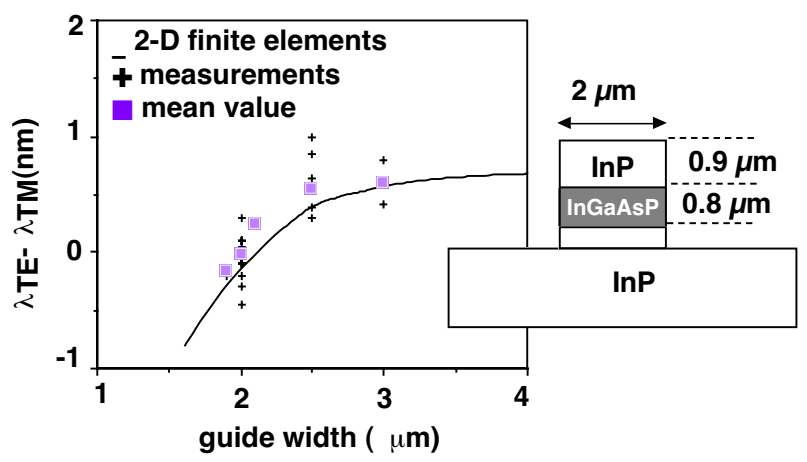

Figure 44. Polarization sensitivity and guide width [Bissessur 96].

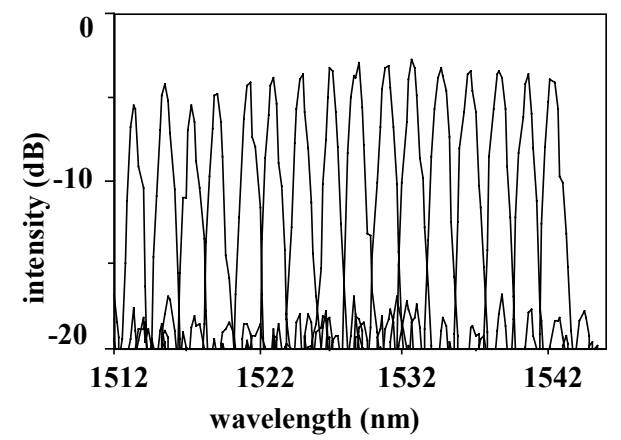

Figure 45. - Measured spectral response of an extremely small 16-channel WDM. Light is coupled into one input guide and the output spectrum is measured for each of the 16 output waveguides. The 16 spectra are shown overlaid [Bissessur 96].

Table VI. 4-channel Wavelength Demultiplexer [Bissessur 95a].

\begin{tabular}{|c|c|}
\hline Wavelength spacing $\Delta \lambda(\mathrm{nm})$ & 2 \\
\hline Crosstalk $(\mathrm{dB})$ & 14 \\
\hline Propagation loss $(\mathrm{dB}), 1$ channel & $5-6$ \\
\hline Fiber to fiber insertion loss $(\mathrm{dB})$ & 14 \\
\hline Birefringence $(\mathrm{nm}) @ 1550 \mathrm{~nm}$ & $\approx 0$ \\
Birefringence $(\mathrm{nm}) @ 1310 \mathrm{~nm}$ & 0.05 \\
\hline
\end{tabular}

Table VII. 16-channel Wavelength Demultiplexer [Bissessur 96].

\begin{tabular}{|c|c|}
\hline Wavelength spacing $\Delta \lambda(\mathrm{nm})$ & 2 \\
\hline Crosstalk $(\mathrm{dB})$ & 14 \\
\hline Propagation loss $(\mathrm{dB}), 1$ channel & $5-6$ \\
\hline Fiber to fiber insertion loss $(\mathrm{dB})$ & $7-8$ \\
\hline Birefringence $(\mathrm{nm}) @ 1550 \mathrm{~nm}$ & $\approx 0$ \\
\hline
\end{tabular}



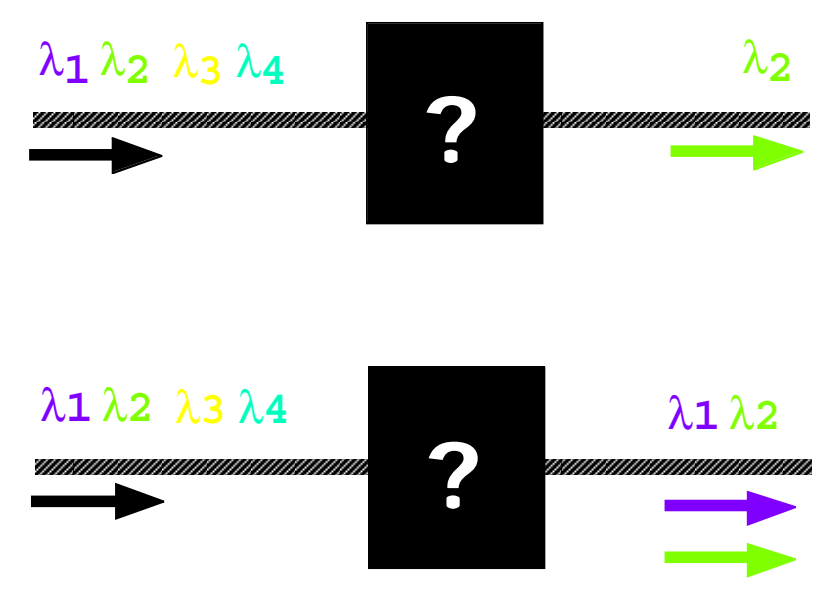

Figure 46. The wavelength selector.

\section{WAVELENGHT SELECTOR}

\subsection{Introduction}

In Wavelength Division Multiplexing systems (WDM), and more specifically for switching of optical packets, extraction (Fig. 46) of one/several channels (one/several wavelengths), requires selective filters with high speed switching time. The wavelength selector combines Wavelength DeMultiplexers (WDM) with fast switching Semiconductor Optical Amplifier (SOA) gates. Furthermore, coupling and propagation losses can be compensated by the SOAs. Low polarization sensitivity is obtained using near zero birefringence WDMs and polarization-independent SOAs. High performances obtained with this device makes it very attractive for WDM systems.

\subsection{Wavelength Selector operating principle}

The wavelength selector (Fig. 47) consists [Mestric 96, Mestric 99] of a first Wavelength DeMultiplexer (WDM1), a SOA gate array and a second identical Wavelength DeMultiplexer $\left(\mathrm{WDM}_{2}\right)$.

Let us assume that we want to select signal at wavelength $\lambda_{1}$ among the 4 multiplexed signals $\lambda_{1}, \lambda_{2}, \lambda_{3}, \lambda_{4}$. The 4 input signals at wavelengths $\lambda_{1}, \lambda_{2}, \lambda_{3}, \lambda_{4}$ are demultiplexed by $\mathrm{WDM}_{1}$. Gate 2 is opened (current is injected in $\mathrm{SOA}_{2}$ ) in order to select the required wavelength $\lambda_{2}$ while gates 1,3 , and 4 are kept closed (no current in the corresponding SOAs). The signal at $\lambda_{2}$ is transmitted to the single output through $\mathrm{WDM}_{2}$. By opening several gates, it is possible to select several gates simultaneously. Since Wavelength selection is made by opening SOA gate, the switching is fast and speed is limited only by the rise and fall times of the SOAs which are typically $<1$ ns [Fernier 92]. 


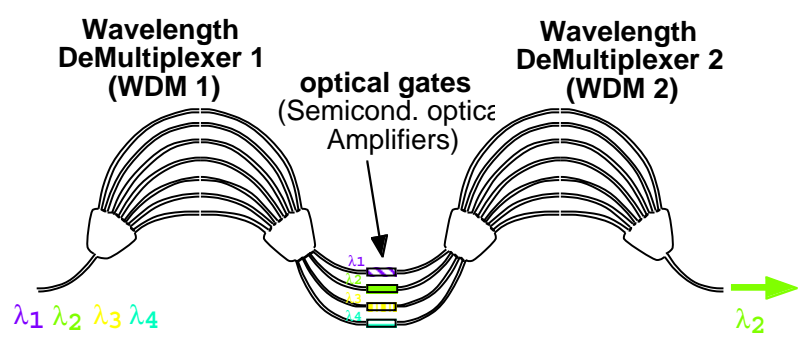

Figure 47. The wavelength selector operating principle.

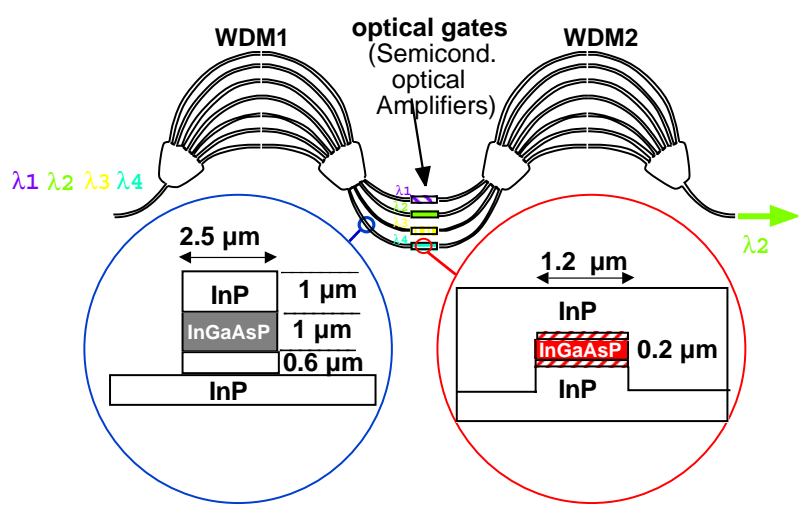

Figure 48. - Waveguide structures of the wavelength selector. The passive waveguide is a deep ridge structure optimized for zero birefringence. The tensile-strained bulk SOA is a Buried Stripe Structure (BRS). Passive and SOA waveguides are butt-join coupled.

\subsection{Wavelength Selector structure and characteristics}

The passive waveguides of the two Wavelength DeMultiplexers consist of a deep ridge structure (Fig. 48) optimized for zero birefringence. For this combination of geometry and layer composition, TE and TM modes have the same effective index, leading to zero birefringence.

Each phase array is made of 30 waveguides with $50 \mu \mathrm{m}$ curvature radius in order to obtain a small $(2.4 \mathrm{~mm} \times 1.2 \mathrm{~mm})$ device. The channel frequency spacing is $400 \mathrm{GHz}(3.2 \mathrm{~nm})$ and the wavelength channels are $\lambda_{1}=1551.4 \mathrm{~nm}, \lambda_{2}=1554.6 \mathrm{~nm}$, $\lambda_{3}=1557.8 \mathrm{~nm}, \lambda_{4}=1561.0 \mathrm{~nm}$. The tensile-strained bulk SOAs (Fig. 48) used for the gates (see "Semiconductor Optical Amplifier" section for details) provide polarization-independent gain to compensate optical losses ( $22 \mathrm{~dB}$ internal gain for $50 \mathrm{~mA}$ current, $550 \mu \mathrm{m}$ length).

The total loss $(19 \mathrm{~dB})$ of the passive sections originates from the phase array $(14 \mathrm{~dB})$ and coupling loss $(5 \mathrm{~dB})$ with the fibers. The passive (WDMs) and active (SOAs) waveguides are butt-join coupled with an estimated $3 \mathrm{~dB}$ coupling loss. This leads to $22 \mathrm{~dB}$ fiber-to-fiber insertion loss. With $50 \mathrm{~mA}$ current in the SOA, the 
fiber-to-fiber insertion loss of the wavelength selector is compensated by the gain provided by the SOAs, allowing overall loss-less operation. The crosstalk (ratio between the selected wavelength signal and remaining signals) is $40 \mathrm{~dB}$.

\subsection{System performances}

Compared to back-to-back transmission, $0.6 \mathrm{~dB}$ and $1.5 \mathrm{~dB}$ sensitivity penalties are obtained respectively at $2.5 \mathrm{~Gb} / \mathrm{s}$ and $10 \mathrm{~Gb} / \mathrm{s}$ for a $10^{-9}$ Bit Error Rate.

\section{TRANSMITTER RECEIVER DEVICE (TRD)}

\subsection{Introduction}

Low cost bi-directional Transmitter-Receiver-Devices (TRD) are required for interactive Fiber-To-The-Home (FTTH) networks (Fig. 49) such as Full Services Access Networks [FSAN]. This target can be reached with monolithic integration of high performance Multi Quantum Well laser with a photodiode, using well established industrial technology. An extensive review of Photonic Integrated Circuits (PIC) modules is given in [Mallecot 2000]. I will report only on the TRD developed in TANIA RNRT project [TANIA].

\subsection{The integrated Transmitter Receiver Device}

Such an integrated device (Fig. 50) must be able to emit at wavelength $\lambda_{1}=1.30 \mu \mathrm{m}$ and simultaneously (full-duplex operation) to detect downstream signal $\lambda_{2}=1.55 \mu \mathrm{m}$ [Mallecot 99]. Upstream (from the Optical Network Unit, ONU at the end user to the Optical Line Termination, OLT) and downstream (from the OLT to the ONU) bit rates are respectively $155.52 \mathrm{Mb} / \mathrm{s}$ and $155.52 / 622.08 \mathrm{Mb} / \mathrm{s}$ as agreed within FSAN and ITUT G. 983. The difficult target specifications are mainly the low Bit Error Rate (BER < $10^{-10}$ at $-33 \mathrm{dBm}$ ), the implicit required low optical crosstalk in full-duplex and low cost. An analysis on the standardization of ONU is reported in [Mallecot 2000].

The integrated device (Fig. 51) consists of a Strained-Layer Multi-Quantum Well (SL-MQW) DFB laser emitting at $\lambda_{1}=1.30 \mu \mathrm{m}$, a $1.55 \mu \mathrm{m}$ photo-detector (bulk GaInAs PIN photodiode) and an isolation (absorption) section (same vertical structure as the laser section) to prevent absorption of the $1.30 \mu \mathrm{m}$ signal by the detector. Strong attenuation of the $1.30 \mu \mathrm{m}$ signal by the isolation section is obtained thanks to reverse biasing the central section and detuning of the lasing wavelength ( $\lambda_{1}=1.30 \mu \mathrm{m}$ is controlled by the grating period) relative to the equivalent band gap wavelength $\left(\lambda_{\mathrm{g}}=1.31 \mu \mathrm{m}\right)$ of the absorbing section.

The downstream signal $\left(\lambda_{2}=1.55 \mu \mathrm{m}\right)$ is absorbed and detected by the photodiode section and guided with low attenuation by the laser and absorption sections due to their larger band gap. The front facet is anti-refection coated $(R=5 \%$ at $\left.\lambda_{2}=1.55 \mu \mathrm{m}\right)$ to improve the input signal coupling. Electrical isolation between laser and detector is ensured by etching almost down to the waveguides. 


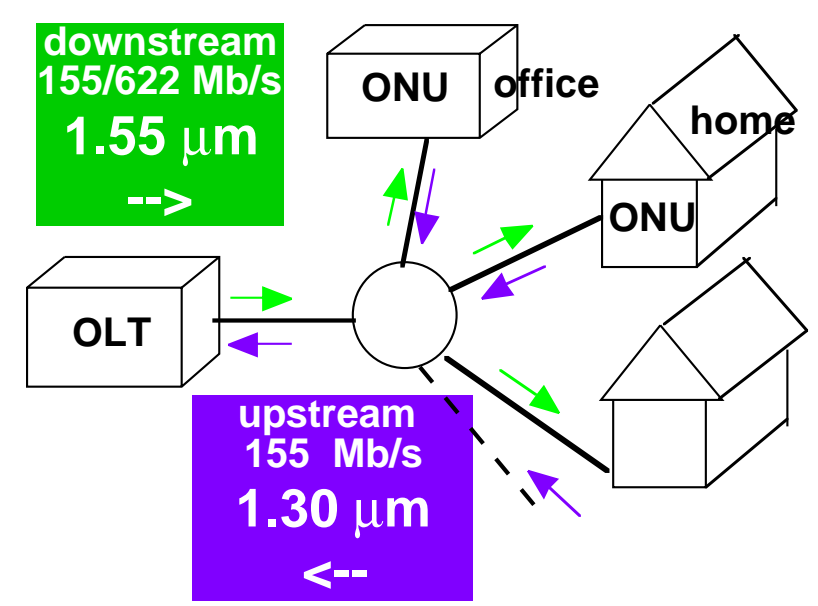

Figure 49. - The laser in the Optical Unit (ONU) emits at $1.30 \mu \mathrm{m}$ (upstream) at $155 \mathrm{Mb} / \mathrm{s}$ towards the Optical Line Termination (OLT). The OLT emits at $1.55 \mu \mathrm{m}(155$ or $622 \mathrm{Mb} / \mathrm{s})$ towards the ONUs. Downstream is detected at $1.55 \mu \mathrm{m}$ by the ONUs.

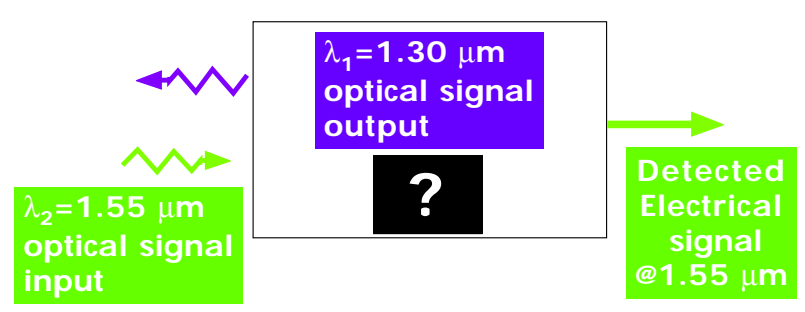

Figure 50. The integrated Transmitter Receiver Device (TRD).

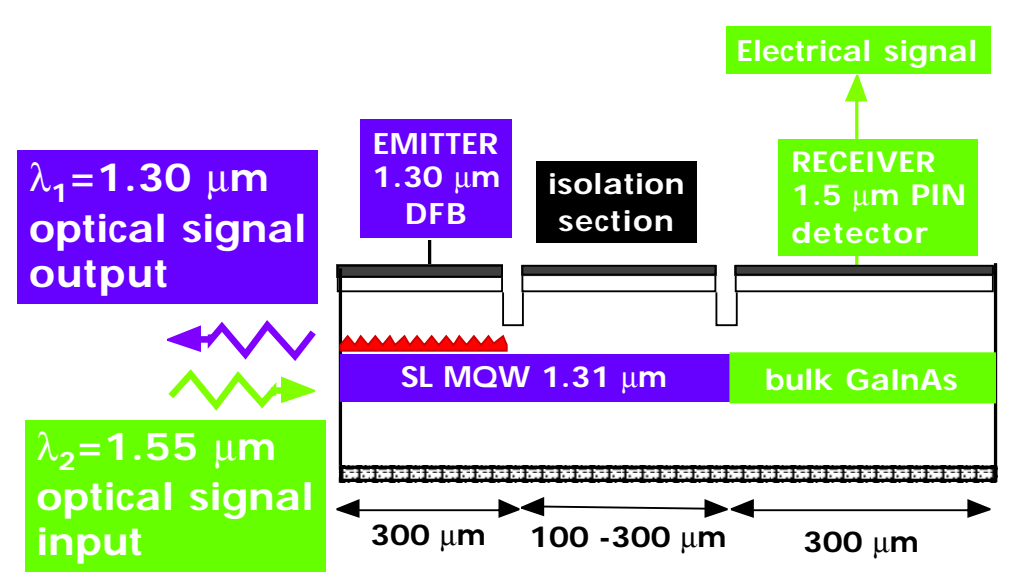

Figure 51. The Transmitter Receiver Device operating principle. 


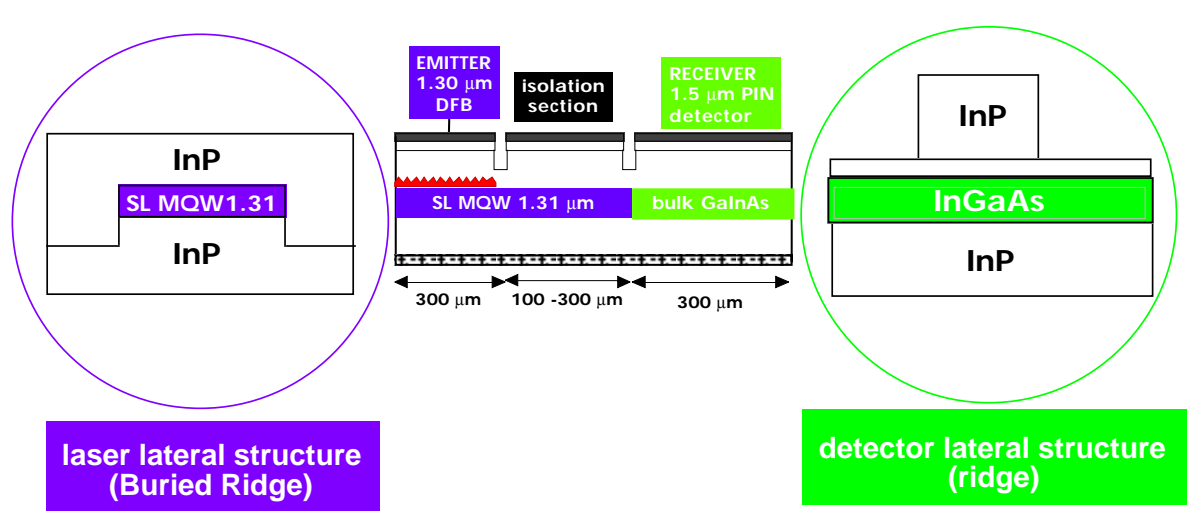

Figure 52. - The Transmitter Receiver Device structure. The laser and detector waveguides are butt-join coupled.

The lateral structures of the laser and detector are shown in the insert of Fig. 52. The lateral current and optical confinements in the laser section are obtained with a Buried Ridge Structure (BRS) with high performances at high temperature [Fernier 95]. The threshold current and external efficiency are respectively $7-27 \mathrm{~mA}$ and $0.32-0.16 \mathrm{~W} / \mathrm{A}$ in the range $0-70{ }^{\circ} \mathrm{C}$. The detector is a ridge structure with $0.3 \mathrm{~A} / \mathrm{W}$ sensitivity, low dark current $(50 \mathrm{nA}$ at $-1 \mathrm{~V})$. The ridge structure is preferred to take advantage of its intrinsic (small size) lower capacitance $(0.5 \mathrm{pF}$ at $-1 \mathrm{~V})$. The laser and detector waveguides are butt-join coupled. Crosstalk must be considered carefully. Even with $\lambda_{1}=1.30 \mu \mathrm{m}<\lambda_{\mathrm{g}}$, with a bad design device, the low energy side of the spontaneous emission spectrum of the emitter can reach the detector, leading to optical crosstalk.

\subsection{System performances}

Full-duplex operation at $155 \mathrm{Mb} / \mathrm{s}$ with $-31 \mathrm{dBm}$ sensitivity has been demonstrated over $20-70^{\circ} \mathrm{C}$ [Mallecot 99]. The extra penalty in full-duplex is as low as $1.5 \mathrm{~dB}$. Coaxial TO packaging of the TRD (connectorized module) is under development [TANIA].

\section{SOURCE FOR WAVELENGTH DIVISION MULTIPLEXING (WDM)}

\subsection{Introduction}

Multiplexing is widely used to increase the capacity (total information bit rate) of transmission systems [Brillouet 98]. Multiplexing can be achieved in the time domain, this is Time Division Multiplexing (TDM) or/and in the spectral domain, this is Wavelength Division Multiplexing (WDM). TDM can be either Electric Time Division Multiplexing (ETDM), or Optical Time Division Multiplexing (OTDM). For example $40 \mathrm{~Gb} / \mathrm{s}$ optical pulses can be obtained by ETDM (Fig. 53) interleaving four 


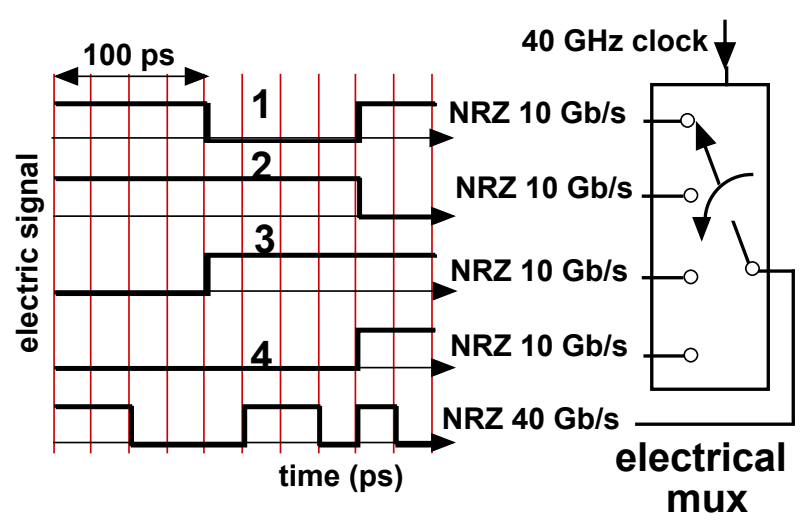

Figure 53. - Electric Time Division Multiplexing (ETDM). Four electric No Return to Zero (NRZ) signals at $10 \mathrm{~Gb} / \mathrm{s}$ are interleaved into a single signal at $40 \mathrm{~Gb} / \mathrm{s}$. Due to limitations of available multiplexer, two-stage multiplexer is necessary to multiplex at this bit rate.

$10 \mathrm{~Gb} / \mathrm{s}$ electrical signals followed by electric-optic conversion or OTDM (Fig. 54), interleaving four $10 \mathrm{~Gb} / \mathrm{s}$ optical streams.

With these techniques, important gain in total information bit rate can be obtained without increasing the actual modulation bit rate of the devices and therefore with a moderate cost increase. WDM will be/is used alone for total information bit rate lower than $1 \mathrm{~Tb} / \mathrm{s}$ and a combination of WDM and TDM for higher bit rates. We will focus on sources for WDM.

\subsection{Wavelength Division Multiplexing}

With Wavelength Division Multiplexing, the basic bit rate is multiplied by the number of channels to take advantage of the bandwidth (1530-1560 nm) of the Erbium Doped Fiber Amplifier.

With the $100 \mathrm{GHz}(0.8 \mathrm{~nm})$ ITU standard channel spacing, $\approx 40$ channels can be transmitted over the same fiber. With Dense Wavelength Division Multiplexing $(50 \mathrm{GHz})$, the number of channels is twice.

The price for this improvement is a severe requirement on sources for WDM: a precise wavelength control better than $0.1 \mathrm{~nm}(\ll 0.8 \mathrm{~nm})$ and a long term wavelength stability. Wavelength stabilization is required to lock the laser to the correct channel. Figure 55 shows an example of WDM with 4 wavelength channels. A $320 \mathrm{~Gb} / \mathrm{s}$ WDM transmission over $6150 \mathrm{~km}$ (dispersion shifted fiber) has been demonstrated [Gautheron 99a] in lab and illustrates the performances that can be obtained. The basic bit rate of the 32 external modulated lasers was $10 \mathrm{~Gb} / \mathrm{s}$, with $50 \mathrm{GHz}$ channel spacing and $50 \mathrm{~km}$ EDFA spacing. A $1.5 \mathrm{~Tb} / \mathrm{s}$ WDM transmission of 150 channels at $10 \mathrm{~Gb} / \mathrm{s}$ over $4 \times 100 \mathrm{~km}$ has also been demonstrated [Bigo 99]. 


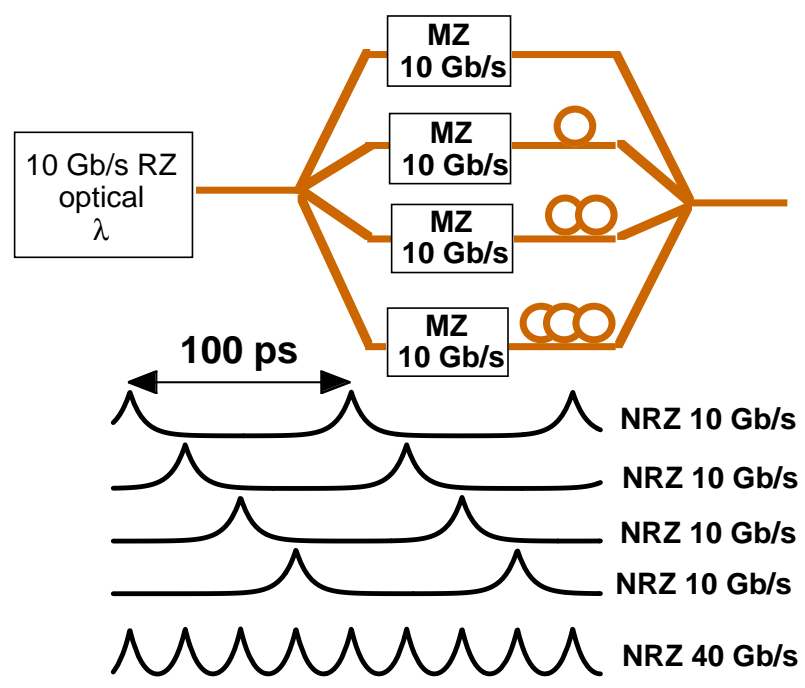

Figure 54. - Optical Time Division Multiplexing (OTDM). Four optical NRZ signals at $10 \mathrm{~Gb} / \mathrm{s}$ are interleaved into a single optical signal at $40 \mathrm{~Gb} / \mathrm{s}$. The RZ $10 \mathrm{~Gb} / \mathrm{s}$ optical signal can be obtained with an Integrated Laser Modulator electrically modulated at $10 \mathrm{GHz}$ at a strong modulation index. A repetition of " 1 " is represented for clarity. The signals are $25,50,75$ ps delayed with fiber delay lines $(20 \mathrm{~cm}$ propagation in standard optical fiber corresponds to $1 \mathrm{~ns}$ delay).

\section{Wavelength multiplexer}

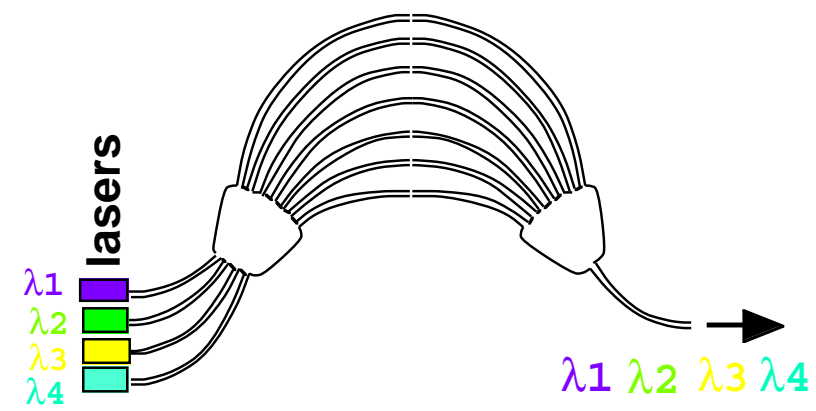

Figure 55. - An example of WDM with 4 wavelength channels. The lasers can be Distributed FeedBack lasers (see Fig. 56), directly modulated at $2.5 \mathrm{~Gb} / \mathrm{s}$. The information bandwidth $(10 \mathrm{~Gb} / \mathrm{s})$ is fourfold increased. Using $10 \mathrm{~Gb} / \mathrm{s}$ Integrated Laser Modulator (ILM), the information bandwidth is $40 \mathrm{~Gb} / \mathrm{s}$. 
Acatel 1915 LMI

$1.55 \mu \mathrm{m}$ digital laser module with optical isolator WDM direct modulation

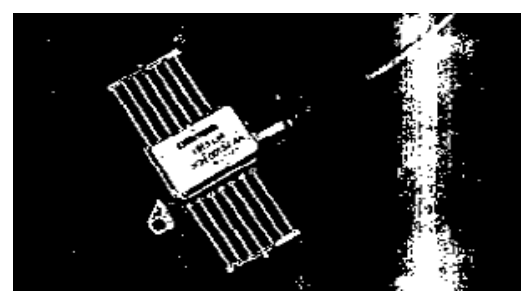

(a)

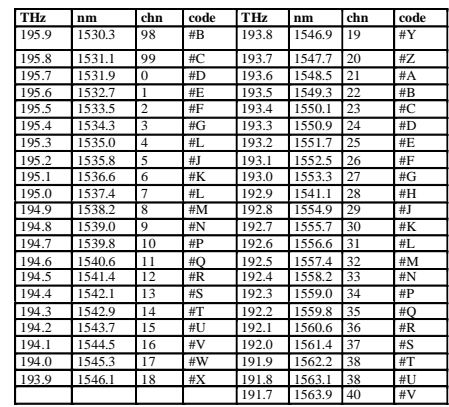

Description

This laser module contains an Alcatel SL-MQW DFB laser with $25 \Omega$ impedance matching designed for use in Wavelenght Division Multiplexed (WDM), direct modulation applications up to $2.5 \mathrm{Gbit} / \mathrm{s}$. The module incorporates a thermo-electric cooler, precision NTC thermistor, and optical isolator for stable operation under all conditions.

\author{
Applications \\ STM-16 and OC-48 \\ synchronous high speed WDM \\ digital transmission systems \\ WDM submarine terminal \\ digital transmission systems \\ Instrumentation
}

(b)

Figure 56. - DFB laser for WDM (From Alcatel Optronics datasheet). (a) Multi-Quantum DFB lasers with fixed wavelengths over the whole ITU range for WDM, suitable for $2.5 \mathrm{~Gb} / \mathrm{s}$ direct modulation. (b) Characteristics.

\title{
9.3. Lasers with fixed wavelength, laser arrays, low cost lasers
}

Multi-Quantum Well DFB lasers with fixed wavelengths over the whole ITU range for WDM application are standard commercial products available at Alcatel Optronics (Fig. 56) for direct modulation (2.5 Gb/s current modulation).

Tunable DBR lasers [Delorme 96] can be integrated in arrays (Fig. 57). Each laser emits at a specific wavelength that can be tuned $(10 \mathrm{~nm})$ by injecting current in the Bragg section. The structure allows 4 different wavelengths with a single pitch grating and $29 \mathrm{~nm}$ overall wavelength range.

Figure 58a shows a low cost WDM source which consists of a standard FabryPerot laser coupled to an external fiber grating [Bissessur 99]. The grating is written in an optical fiber and the Bragg wavelength is determined by the grating pitch. The bandwidth of the Bragg reflector is $<0.1 \mathrm{~nm}$ in order to control the wavelength within $1 / 10$ of the $0.8 \mathrm{~nm}(100 \mathrm{GHz})$ ITU wavelength spacing. Any wavelength of the ITU grid can be obtained by using the same FP laser and a convenient fiber grating 


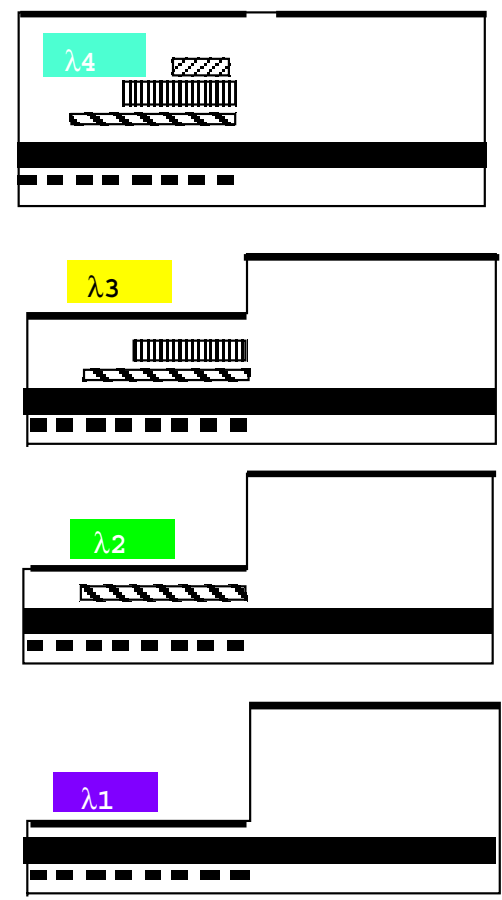

Figure 57. - 4-wavelength tunable DBR laser array [Delorme 96]. With a single grating pitch, the Bragg wavelength is different in each laser since the Bragg sections have a different transverse structure. Each Bragg wavelength can be tuned $(10 \mathrm{~nm})$ around its nominal value through current injection. Overall wavelength tuning range is $29 \mathrm{~nm}$.

pitch $\Lambda$ (Fig. 58b). With a $2 \mathrm{~cm}$ external cavity length, the round trip frequency $c /(2 n L)$ is $5 \mathrm{GHz}$, allowing $2.5 \mathrm{~Gb} / \mathrm{s}$ direct modulation. $10 \mathrm{~Gb} / \mathrm{s}$ requires an external cavity shorter than $1 \mathrm{~cm}$.

Thermal stability of the Bragg wavelength is mainly determined by the weak temperature dependence of the refractive index of the fiber $\left(\mathrm{d} \lambda / \mathrm{d} T=0.01 \mathrm{~nm} /{ }^{\circ} \mathrm{C}\right)$, an order of magnitude lower than in semiconductor lasers. The laser is a Multi-Quantum Well (MQW) Buried Ridge structure with mode-expanding taper to improve the coupling with the fiber. $2.5 \mathrm{~Gb} / \mathrm{s}$ transmission over $180 \mathrm{~km}$ of standard fiber has been demonstrated in the 1540-1560 $\mathrm{nm}$ range, and $10 \mathrm{~Gb} / \mathrm{s}$ with a short external cavity (8 $\mathrm{mm}, 12 \mathrm{GHz}$ roundtrip frequency) [Bissessur 99].

\subsection{Tunable lasers}

A tunable laser can be used as a spare in WDM systems, avoiding having a spare for each wavelength. Wavelength tuning is obtained by injecting current in the passive Bragg section of a Distributed Bragg Reflector (DBR) laser (Fig. 59, [Delorme 95]). Increasing the carrier density decreases the refractive index and consequently decreases 


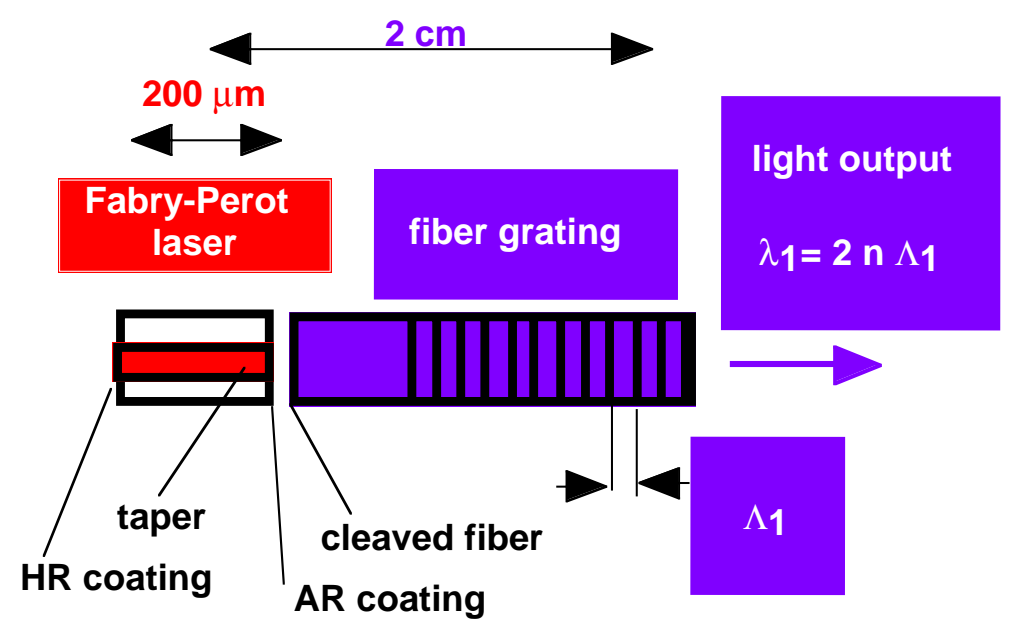

(a)

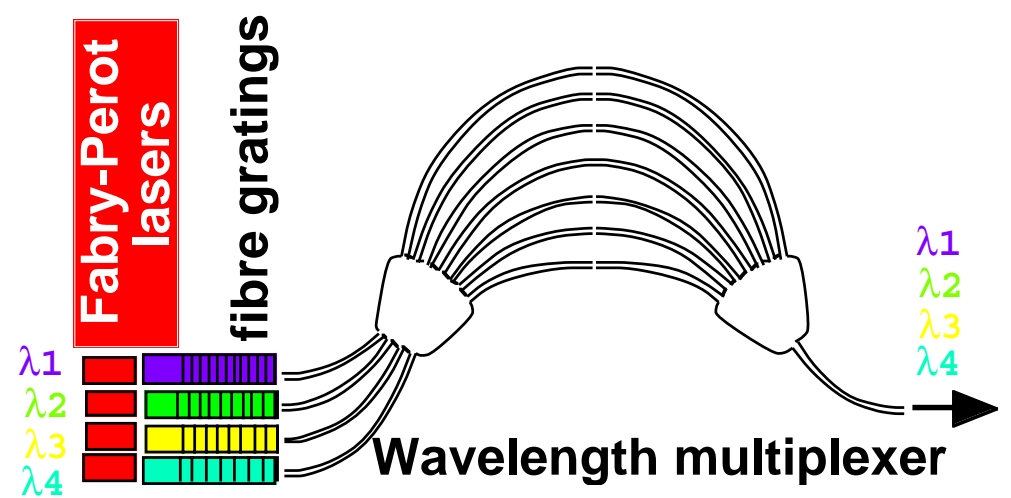

(b)

Figure 58. — External grating fiber laser [Bissessur 99]. (a) external grating fiber laser which consists of a standard low cost Fabry-Perot laser coupled to an external fiber grating. $\Lambda_{1}$ is the grating pitch, $n$ the refractive index of the fiber $(\approx 1.5)$ and $\lambda_{1}$ the Bragg wavelength. Any wavelength of the ITU grid can be obtained by using the same FP laser and a convenient fiber grating pitch $\Lambda$; (b) 4 external grating fiber lasers with different wavelengths and same Fabry-Perot laser. The four different Bragg wavelength are obtained thanks to different grating pitch $\Lambda_{1}, \Lambda_{2}, \Lambda_{3}, \Lambda_{4}$. See details of the external grating fiber lasers in Figure 58a.

the Bragg wavelength. The tuning range is of the order of 5-15 nm. The wavelength can also be tuned by heating $\left(\approx 0.1 \mathrm{~nm} /{ }^{\circ} \mathrm{C}\right)$ controling the temperature.

Wider (100 nm) tuning range (Fig. 60) can be obtained with complex DBR laser such as the Coupler Sampled Reflector laser (GCSR) [Rigole 95]. The coupler section ensures coarse wavelength tuning and the Bragg and phase sections fine tuning. The wavelength is controlled using a microprocessor and a stored mapping of the wavelengths and associated currents. 


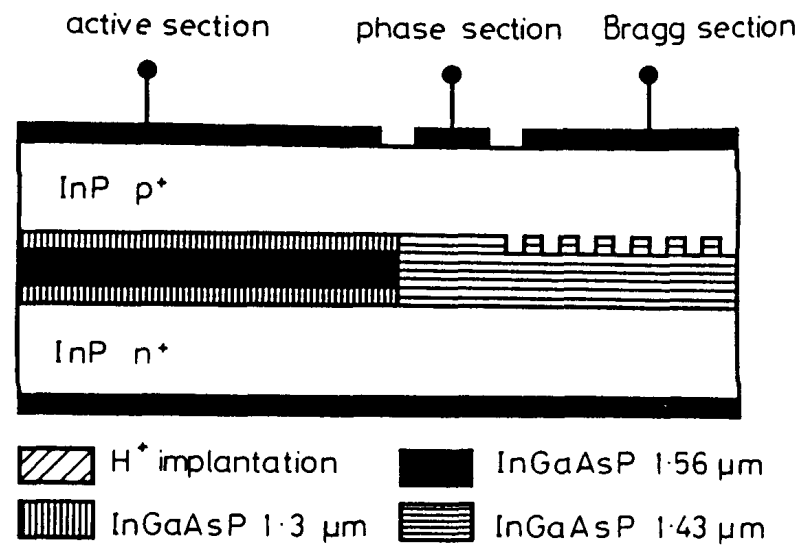

Figure 59. - Butt-join tunable (15 nm range) Distributed Bragg Reflector laser [Delorme 95]. The lasing wavelength is adjusted thanks to the current injected in the Bragg and phase sections. Three MOVPE seps are required to grow this BRS structure. Wavelength Tuning range is $15 \mathrm{~nm}$.

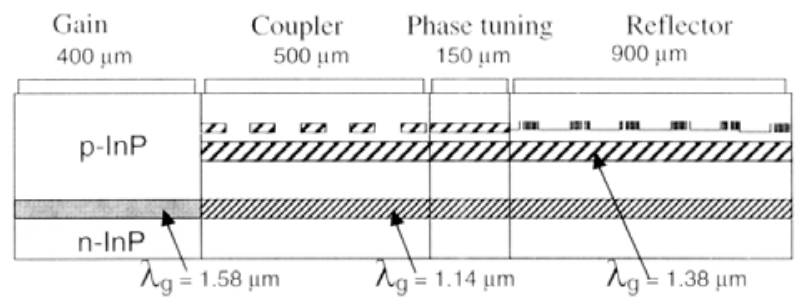

Figure 60. - Grating Coupler Sampled Reflector laser (GCSR) [Rigole 95]. The coupler section (15 $\mu \mathrm{m}$ period) ensures coarse wavelength tuning and the Bragg and phase sections fine tuning. Five epitaxial steps are required to grow this structure. Wavelength Tuning range is $100 \mathrm{~nm}$.

\section{Acknowledgements}

I wish to thank my colleagues at OPTO+ who make the devices, Pierre Chavel, JeanPaul Pocholle, Jean-Claude Saget, Claude Fabre, SFO and CNRS, the organizers of this summer school. 


\section{References}

[Adams 96] D.M. Adams, C. Rolland, N. Puetz, R.S. Moore, F.R. Shepherd, H.B. Kim and S. Bradshaw, "Mach-Zehnder modulator integrated with a gain-coupled DFB laser for $10 \mathrm{Gbit} / \mathrm{s}, 100 \mathrm{~km}$ NDSF transmission at $1.55 \mu \mathrm{m}$ ", Electron. Lett. 32 (1996) 485-486.

[Alferov 70] Zh.I. Alferov, V.M. Andreev, D.Z. Garbuzov, Yu V. Zhilyaev, E.P. Morozov, E.L. Portnoi and V.G. Trofim, "Investigation of the influence of the AlAs-GaAs heterostructure parameters on the laser threshold current and the realization of continuous emission at room temperature", Sov. Phys. Semicond. 4 (March 1971), 1573-1575 [Translated from Fiz. Tekh. Poluprovodn 4 (1970)] 1826.

[Bigo 99] S. Bigo et al., "1.5 Tb/s WDM transmission of 150 channels at $10 \mathrm{~Gb} / \mathrm{s}$ over 4x100 km of TeraLightTM fiber", Post deadline paper, ECOC'99 (1999).

[Bissessur 95a] H. Bissessur, F. Gaborit, B. Martin, G. Ripoche, "Polarisationindependent phased-array demultiplexer on InP with high fabrication tolerance", Electron. Lett. 31 (1995) 1372-1373.

[Bissessur 95b] H. Bissessur, B. Martin, R. Mestric, F. Gaborit "Small-size polarisation-independent phased-array demultiplexers on $\mathrm{InP}^{\prime \prime}$, Electron. Lett. 31 (1995) 2118-2120.

[Bissessur 96] H. Bissessur, P. Pagnod-Rossiaux, R. Mestric, B. Martin, “Extremely small polarisation independent phased-array demultiplexers on InP", IEEE Photon. Technol. Lett. 8 (1996).

[Bissessur 99] H. Bissessur, C. Caraglia, B. Thedrez, J. M. Raisant, I. Riant, "Wavelength-versatile external fiber grating lasers for $2.5 \mathrm{~Gb} / \mathrm{s}$ WDM networks", IEEE Photon. Technol. Lett. 11 (1999) 1304-1306.

[Brillouet 98] F. Brillouet, F. Devaux, M. Renaud, "From transmission to processing: challenges for new optoelectronic", Alcatel Telecommunications Review (1998) 232240.

[Brosson 94] P. Brosson, "Analytical model of a semiconductor optical amplifier", IEEE J. Lightwave Technol. 12 (1994) 49-54.

[Brosson 96] P. Brosson and H. Bissessur, "Analytical expressions for the FM and AM responses of an Integrated Laser-Modulator", IEEE J. Selected Topics In Quantum Electron. on Integrated Optics 2, (1996) 336-340.

[Brosson 97] P. Brosson and A. Lestra, "Numerical simulation of an Integrated Laser Modulator", invited paper, TU-3-1 -SBMO/IEEE International Microwave Symposium, Natal, Brazil (1997).

[Brosson 98a] P. Brosson, S.C. Auzanneau, H. Helmers, J.G. Provost, P. Delansay, "Optical feedback modeling in integrated Laser Mach-Zehnder modulator: application to frequency chirp monitoring by AM measurements", 16th IEEE International Semicond. Laser Conf., Nara, Japan, TuE30 (1998).

[Brosson 98b] P. Brosson, P. Delansay, "Modeling of the static and dynamic responses of an Integrated Laser Mach-Zehnder modulator and comparison with an Integrated Laser E/A modulator", IEEE J. Lightwave Technol. 16 (1998) 2407-2418. 
[Chiaroni 93] D. Chiaroni, P. Gavignet-Morin, P.A. Perrier, S. Ruggeri, S. Gauchard, D. De Bouard, J.C. Jacquinot, C. Chauzat, J. Jacquet, P. Doussière, M. Monnot, E. Grard, D. Leclerc, M. Sotom, J.M. Gabriagues, J. Benoit, “Rack-Mounted 2.5 Gbit/s ATM photonic Switch Demonstrator", ECOC'93, Postdeadline paper, ThP 12.7, Montreux (1993).

[Chiaroni 97] D. Chiaroni et al., "New $10 \mathrm{~Gb} / \mathrm{s} 3-\mathrm{R}$ NRZ optical regenerative interface based on semiconductor optical amplifiers for all-optical networks", Postdeadline paper, ECOC'97, Edinburg, UK (1997) 41-43.

[Dagens 99] B. Dagens, D. Leclerc, V. Verdrager, F. Poingt, I. Guillemeot, F. Gaborit, D. Ottenwalder, "Design Optimization of all-active Mach-Zehnder wavelengths converters", IEEE Photon. Technol. Lett. (1999) 424-426.

[Delansay 97] Ph. Delansay, D. Penninckx, E. Boucherez, C. Fortin, O. Le Gouezigou, "InP/InGaAsP phase-shifted Mach-Zehnder modulator for wavelengthindependent (1530-1560 $\mathrm{nm}$ ) performance in $10 \mathrm{Gbit} / \mathrm{s}$ transmission over dispersive fiber", OFC'97, TuD1 (1997).

[Delansay 98] Ph. Delansay, S. Gauchard, H. Helmers, D. Penninckx, S. Gurib, F. Brillouet, "2.5 Gb/s transmission over $1086 \mathrm{~km}$ of standard single-mode fiber using an Integrated Laser Mach-Zehnder modulator", OFC'98, TuI7 (1998).

[Delorme 95] F. Delorme, et al., "Butt-jointed DBR laser with $15 \mathrm{~nm}$ tunability, grown in three MOVPE steps", Electron. Lett. 31 (1995) 1244.

[Delorme 96] F. Delorme, et al., "4-wavelength DBR laser array fabricated with a single grating holographic exposure technique", OFC'96, FB3, (1996) 300.

[Dorgeuille 2000] F. Dorgeuille, L. Noirie, J.P. Faure, A. Ambrosy, S. Rabaron, F. Boubal, M. Schilling, C. Artigue, «1.28 Tbit/s throughput $8 \times 8$ optical switch based on Gain-Clamped Semiconductor Optical Amplifier gates », OFC'2000.

[Doussière 92a] P. Doussière et al., "Polariation-insensitive semiconductor optical amplifier with buried laterally tapered active waveguide", Optical amplifiers and their applications, Santa Fe (1992).

[Doussière 92b] P. Doussière, P. Garabedian, V. Colson, O. Legouezigou, F. Leblond, J.L. Lafragette, M. Monnot, B. Fernier, "Polarisation insensitive semiconductor optical amplifier with lateraly tapered active waveguide", 3rd Top. Meet. on Optical Amplifiers and their Applications, FA2, Santa Fe, New Mexico, USA (1992).

[Doussière 94a] P. Doussière, P. Garabedian, C. Graver, D. Bonnevie,T. Fillion, E. Derouin, M. Monnot, J.G. Provost, D. Leclerc, M. Klenk, “1.55 $\mu \mathrm{m}$ polarisation independent semiconductor optical amplifier with 25 dB fiber to fiber gain", IEEE Photon. Technol. Lett. 6 (1994) 170-172.

[Doussière 94b] P. Doussière, 14th IEEE Semiconductor Laser Conf. (1994).

[Duan 93] G. Duan et al., "1 Gb/s operation of optically triggered MQW bistable lasers incorporating a proton bombarded MQW bistable lasers incorporating a proton bombarded absorber", CLEO'93, Baltimore (1993).

[Durhuus 93] T. Durhuus, C. Joergensen, B. Mikkelsen, K.E. Stubkjaer, "Penalty free all-optical wavelength conversion by SOA's in Mach-Zehnder configuration", ECOC'93, Montreux (1993) 129. 
[Erman 95] M. Erman, "Prospects for Integrated Optics in Telecom Applications", ECIO'95, Delft, NL (1995).

[Erman 96] M. Erman, "Semiconductor based photonic integration for switching", Sendai, Japan (1996).

[Fernier 92] B. Fernier, P. Brosson, D. Bayart, P. Doussière, R. Beaumont, F. Leblond, P. Morin, G. Da Loura, J. Jacquet, E. Derouin, P. Garabedian, “Fast (300 ps) polarization insensitive semiconductor optical amplifier switch with low driving current $(70 \mathrm{~mA})$ ", Proc. 13th IEEE International Semiconductor Laser Conference, Takamatsu (1992) 130-131.

[Fernier 95] B. Fernier, F. Gerard, P. Pagnod, G. Michaud, G. Ripoche, G. Vendrome, R.M. Capella, "'1.3 mm SL-MQW DFB lasers with high quantum efficiency for -40-85 ${ }^{\circ}$ C operation", Electron. Lett. 31 (1995) 2174-2175.

[FSAN] Full Service Access Network (FSAN) requirement specification, Ed. J. Quayle (1997). http://www. fsanet.net/access/issue3.htm

[Gabriagues 91] J.M. Gabriagues, J.B. Jacob, "Exploitation of the wavelength domain for photonic switching in the IBCN", ECOC/IOOC'91, Tech. Dig., invited paper, Paris (1991) 59-66.

[Gabriagues 93] J.M. Gabriagues, "Optical ATM switching techniques", OFC/IOOC'93, Tech. Dig., invited paper, WD1, San Jose (1993).

[Gautheron 99a] O. Gautheron, J.B. Leroy, P. Marmier, WJ3, San Diego, OFC'99 (1999).

[Gautheron 99b] O. Gautheron et al., "32x10 Gb/s transmission over $6150 \mathrm{~km}$ with a $50 \mathrm{GHz}$ wavelength spacing", WJ3-1, OFC'99 (1999).

[Gavignet 95] Gavignet, M. Sottom, J.C. Jacquinot, P. Brosson, D. Leclerc, W. Hunziker, H. Duran, "Penalty-free $2.5 \mathrm{Gbit} / \mathrm{s}$ photonic switching using a semiconductor four-gate array module", Electron. Lett. 31 (1995) 487.

[Haisch 95] H. Haisch, D. Baums, E. Latch, D. Kaiser, K. Satske, J. Weber, R. Weinmann, P. Wiedemann, E. Zielinski, "Record bandwidth (42 GHz measured) and polarisation insensitive $(<0.4 \mathrm{~dB})$ tensile strained InGaAsP MQW ridge waveguide electroabsorption modulator for ultra-high bit rate TDM systems", Proc. 21st Eur. Conf. on Opt. Comm., Th.B.3.1 (1995) 1007-1010.

[Hayashi 70] I. Hayashi, M.B. Panish, P.W. Foy and S. Sumski, “Junction lasers which operate continuously at room temperature", Appl. Phys. Lett. 17 (1970) 109.

[Hunziker 95] W. Hunziker, W. Vogt, H. Melchior, D. Leclerc, P. Brosson, F. Pommereau, R. Ngo, P. Doussière, F. Mallecot, T. Fillion, I. Wamsler, G. Laube " Selfaligned flip-chip packaging of tilted semiconductor optical amplifier arrays on $\mathrm{Si}$ motherboard ", Electron. Lett. 31 (1995) 488.

[Janz 99a] C. Janz, B. Dagens, A. Bisson, F. Poingt, F. Pommereau, F. Gaborit, I. Guillemot, M. Renaud, "Integrated all-active Mach-Zehnder wavelength converter with $-10 \mathrm{dBm}$ signal sensitivity and $15 \mathrm{~dB}$ dynamic range at $10 \mathrm{~Gb} / \mathrm{s}$ ", Electron. Lett. 35 (1999) 588. 
[Janz 99b] C. Janz, F. Poingt, F. Pommereau, W. Grieshaber, F. Gaborit, D. Leclerc, I. Guillemot, M. Renaud, "All-active Dual Order Mode (DOMO) Mach-Zehnder

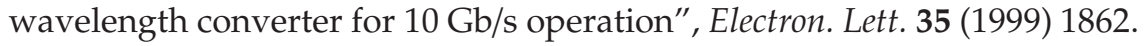

[Janz 99c] C. Janz, B. Dagens, A. Bisson, F. Poingt, F. Pommereau, F. Gaborit, I. Guillemot, M. Renaud, "Integrated all-active wavelength converter with record sensitivity and large dynamic range at 10 Gb/s", FB3-1, OFC'99 (1999).

[Joergensen 92] C. Joergensen, T. Durhuus, B. Fernier, P. Garabedian, E. Derouin, F. Leblond, B. Mikkelsen, K.E. Stubkjaer, "Optically controlled semiconductor optical amplifiers gates", ECOC'92, Berlin (1992).

[Kapron 70] F.P. Kapron, D.B. Keck, R.D. Maurer, "Radiation losses in glass optical waveguides", Appl. Phys. Lett. 17 (1970) 423-425.

[Leclerc 95] D. Leclerc, P. Brosson, F. Pommereau, R. Ngo, P. Doussière, F. Mallecot, P. Gavignet, I.Wamsler, G. Laube, W. Hunziker, W. Vogt, H.Melchior, "High performance semiconductor optical amplifier array for self aligned packaging using Si V-groove flip chip technique", IEEE Photonics Technol. Lett. (1995) 476.

[Lesterlin 96a] D. Lesterlin, S. Artigaud, V. Rodrigues, J.G. Provost, K. Satzke, J.P. Hebert, E. Derouin, G. Michaud, O. Le Gouezigou, D. Penninckx, H. Großkopf, A. Bodéré, J.L. Genter, H. Haisch, J.L. Beylat, J.F. Vinchant, "High performance and reproducible integrated laser-modulator for $10 \mathrm{~Gb} / \mathrm{s}$ NRZ transmission over standard fiber", ECOC'96, Wep.20 (1996) 3.289.

[Lesterlin 96b] D. Lesterlin, S. Artigaud, H. Haish, “Integrated Laser/modulators for 10 Gbit/s systems", ECOC'96, Oslo, Technical Digest, invited paper WeD.3.1 (1996) 3.183.

[Lesterlin 97] D. Lesterlin, S. Artigaud, J.G. Provost, A. Lestra, P. Brosson, O. Le Gouezigou, H. Großkopf, D. Carpentier, G. Michaud, A. Pinquier, H. Haisch, J.F. Vinchant, "76 km transmission over standard dispersion fiber at $10 \mathrm{Gbit} / \mathrm{s}$ using a high power integrated laser-modulator and a PIN receiver without any optical amplifier", OFC'97, WL37 (1997) 199.

[Lestra 96a] A. Lestra, P. Brosson, O. Le Gouezigou, D. Lesterlin, J.G. Provost, “Experimental demonstration of the combined phase and amplitude reflectivity effects on the chirp of a $10 \mathrm{Gbit} / \mathrm{s}$ integrated laser-modulator source", 15th IEEE International Semicond. Laser Conf., Haifa, Israel (1996) W1.2.

[Lestra 96b] A. Lestra, P. Brosson, "Design rules for a low chirp integrated DFB laser with an Electroabsorption modulator", IEEE Photonics Technol. Lett. 8 (1996) 998-1000.

[Mallecot 99] F. Mallecot, H. Nakajima, A. Leroy, A. Plais, C. Chaumont, D. Carpentier, E. Derouin, F. Gaborit, J. Jacquet, "-31 dBm sensitivity of a monolithic TransmitReceive_Device over wide temperature range", ThN2, San Diego, OFC'99 (1999).

[Mallecot 2000] F. Mallecot, F. Doukhan, J. Harari, F. Laune, “Low cost transceivers for FSAN applications", invited paper, NOC (2000).

[Marcuse 90] D. Marcuse, "DFB laser with attached external intensity modulator", IEEE J. Quantum Electron. 26 (1990) 262-269. 
[Mestric 96] R. Mestric, M. Renaud, B. Martin, F. Gaborit, "Démultiplexeurs en longueur d'onde à réseau de guides sur InP", JNOG'96, Nice (1996).

[Mestric 99] R. Mestric, M. Renaud, F. Pommereau, B. Martin, F. Gaborit, C. Janz, I. Guillemot, D. Leclerc, "Loss-less 4-channel wavelength selector monolithically integrated on InP", paper ThB2-1, OFC'99, San Diego (1999).

[Mikkelsen 93a] B. Mikkelsen et al., OFC/IOOC'93, San Jose (1993) 34.

[Mikkelsen 93b] B. Mikkelsen et al., "20 Gbit/s polarisation insensitive wavelength conversion in semiconductor optical amplifiers", ECOC'93, Montreux (1993).

[Ottolenghi 93] P. Ottolenghi, A. Jourdan, J. Jacquet, ECOC'93, Montreux (1993).

[Penninckx 97] D. Penninckx, Ph. Delansay, "Comparison of the propagation performance over standard dispersive fiber between InP-based $\pi$-phase-shifted and symmetrical Mach-Zehnder modulators", IEEE Photon. Technol. Lett. 9 (1997) 12501252.

[Renaud 96] M. Renaud, M. Bachmann, M. Erman, "Semiconductor Optical Space Switches", IEEE J. of Selected Topics in Quantum Electron. 2 (1996) 277.

[Rigole 95] P.J. Rigole et al., "Improved tuning regularity over $100 \mathrm{~nm}$ in a vertical Grating assisted Codirectional Coupler laser with a Super Structure Grating distributed Bragg reflector", Tu A.3.6, ECOC'95 (1995) 219.

[Serre 93] F. Serre et al., "Déclenchement optique à $2.5 \mathrm{~Gb} / \mathrm{s}$ d'un laser à semiconducteur bistable, à absorbeur saturable protonné, sans erreur de transmission", Journées Nationales d'optique guidée, Marseille (1993).

[Simes 92] R. Simes, E. Gaumont-Goarin, C. Labourie, D. Bonnevie, A. Perales, D. Lesterlin, L. Goldstein, "GSMBE growth on patterned substrates for optoelectronic devices”, Tech. Dig. IPRM'92, Newport, USA (1992) 198-201.

[Tanbun-Ek 95] T. Tanbun-Ek, P.F. Sciortino, Jr., A.M. Sergent, K.W. Wetcht, P. Wisk, Y.K. Chen, C.G. Bethea, S.K. Sputz, "DFB lasers integrated with Mach-Zehnder optical modulator fabricated by selective area growth MOVPE technique", IEEE Photon. Technol. Lett. 7, (1995) 1019-1021.

[TANIA] "Terminal d'Accès Numérique InterActif", Réseau National de Recherche en Télécommunication (RNRT) project, http: //www . telecom.gouv . fr/rnrt/projets/ptania.htm

[Tromborg 87] B. Tromborg, H. Olesen, X. Pan, S. Saito, "Transmission line description of optical feedback and injection locking for Fabry-Perot and DFB lasers", IEEE J. Quantum Electron. 23 (1987) 1875-1889.

[Yu 96] J. Yu, C. Rolland, D. Yevick, A. Somani, S. Bradshaw, "Phase-engineered III-V MQW Mach-Zehnder modulators", IEEE Photon. Technol. Lett. 8 (1996) 1018-1020.

[Zucker 92] J.E. Zucker, K.L. Jones, M.A. Newkirk, R.P. Gnall, B.I. Miller, M.G. Young, U. Koren, C.A. Burrus, B. Tell, "Quantum well interferometric modulator monolithically integrated with $1.55 \mu \mathrm{m}$ tunable distributed Bragg reflector laser", Electron. Lett. 28 (1992) 1888-1889. 